\title{
MORFOTECTÓNICA DEL SEGMENTO CÁDIZ-MÁLAGA, SUR DE ESPAÑA
}

\section{MORPHOTECTONICS OF THE CADIZ-MALAGA SEGMENT, SOUTHERN SPAIN}

\author{
Mario Octavio Cotilla-Rodríguez ${ }^{1}$ \\ Diego Córdoba-Barba \\ Universidad Complutense de Madrid, España
}

\section{RESUMEN}

Se presentan los resultados de una investigación morfotectónica del segmento continental de Cádiz a Málaga, zona occidental de interacción entre las placas africana y euroasiática. Aquí existe un sistema de 5 tipos de Unidades Territoriales (macrobloques $=2$, mesobloques $=2$, bloques $=29$, microbloques $=44$, y nanobloques $=202$ ) en relaciones espacio-temporales actuales con una red de 29 alineaciones principales y 32 intersecciones o nudos. El 59\% de los bloques es muy activo o activo, y el territorio tiene un valor de $\sim 0.03$ fracturas $/ \mathrm{km}^{2}$ con 63 escarpes. Las zonas con mayor actividad están entre las localidades de Barbate-Torrecera-Sierra de las Cabras (en Cádiz) y Ronda-Embalse Gudaltela-Cabras-Villalón (en Málaga). El segmento de Cádiz-Málaga tiene características similares a las de la zona colindante de Almería-Córdoba-Granada-Jaén-Murcia. Todo el trabajo sustentado en un SIG.

Palabras clave: Cádiz, España, Málaga, morfotectónica, SIG

1 Doctor en Ciencias Física y Profesor del Departamento de Geofísica y Meteorología, Facultad de Ciencias Físicas. Universidad Complutense de Madrid, Ciudad Universitaria, 28040 Madrid. Correo electrónico: macot@ucm.es

2 Doctor en Ciencias Física y Profesor del Departamento de Geofísica y Meteorología, Facultad de Ciencias Físicas. Universidad Complutense de Madrid, Ciudad Universitaria, 28040 Madrid. Correo electrónico: dcordoba@ucm.es 
Mario Octavio Cotilla-Rodríguez, Diego Córdoba-Barba. Morphotectonics of the

Cadiz-Malaga Segment, Southern Spain

\begin{abstract}
We present the results of a morphotectonic research of the Cadiz to Malaga continental segment, which is a western interaction zone between the African and Europe-Asian plates. There is a system of 5 types of Territorial Units (macroblocks $=2$, mesoblocks $=2$, blocks $=29$, microblocks $=44$, and nanoblocks $=202$ ) in current space-time relations with a network of 29 main alignments and 32 intersections or junctions. Fifty-nine percent of the blocks are very active or active, and the territory has a mean value of $\sim 0.03$ fractures $/ \mathrm{km}^{2}$ with 63 scarps. The most active zones are between Barbate-Torrecera-Sierra de las Cabras (in Cádiz) and Ronda-Embalse Gudaltela-Cabras-Villalon (in Málaga). This Cadiz-Malaga segment has similar characteristics to those determined for the adjacent zone of Almería-Córdoba-Granada-Jaén-Murcia. All data are in a GIS.
\end{abstract}

Keywords: Cadiz, GIS, Malaga, morphotectonics, Spain

\title{
Introducción
}

Una investigación morfotectónica es una tarea de tipo Geográfica que integra resultados de otras ciencias afines; que conlleva, inicialmente, recorrer la región de estudio y la revisión de los resultados en la geología, la tectónica, la geomorfología, la geofísica, la sísmica, la sismicidad, etc. (Cotilla y Córdoba, 2004A). Se usan los datos e ideas principales para una base inicial, estos hay que contrastarlos en el terreno y complementarlos; así se evita la duplicidad de esfuerzos y corrigen deficiencias (Cotilla, Córdoba, y Sánchez, 2003). Esta es una metodología esencialmente rusa (Rantsman, 1979).

Los métodos morfotectónicos permiten distinguir la evolución geotectónica de las morfoestructuras $\mathrm{y}$, valorar los complejos morfoesculturales. Se determinan los elementos tectónicos y las deformaciones de los niveles geomorfológicos; se precisan, cronológica y jerárquicamente, los límites de las Unidades Territoriales [UT], los elementos lineales (alineaciones o morfoalineamientos) y las intersecciones de ellos (o nudos), la cual permite enfrentar problemas geofísicos. Los métodos se han incluido en un Sistema de Información Geográfica (SIG) (Cotilla y Córdoba, 2003); este sistema se ha probado y ajustado en las investigaciones realizadas por los autores en Cuba, España, Jamaica, La Española y Puerto Rico, para ello nos apoyamos en: Alekseevskaya et al. (1977), Assinovskaya y Solovyev (1994), Cotilla y Córdoba (2009, 2007, 2004A, B, 2003, en prensa); Cotilla et al. (2004, 2003), Gatinsky y Rundquist (2004), Gorshkov et al. (2000), Rantsman (1979) y Zhidkov et al. (1975). En los objetivos, está la determinación de zonas con mayor probabilidad de terremotos, y la relación del sistema con los movimientos tectónicos recientes y la actividad sísmica [AS].

170 Revista Geográfica de América Central No 55 ISSN 1011-484X, julio-diciembre 2015, pp. 169-198 


\section{Área de estudio}

La investigación tiene como antecedentes a 7 trabajos de los autores, para otras regiones de la Península Ibérica [PI] (Figura 1A). Aquí estudiamos el segmento de las provincias de Cádiz [C] y Málaga [M] (Figuras $1 \mathrm{~A}$ y B), del S y SW de la Comunidad de Andalucía (Figuras 2A y B). La región tiene una longitud de $\sim 240 \mathrm{~km}$ y superficie de $\sim 87,300 \mathrm{~km}^{2}$. El relieve está definido en el sistema montañoso (Cordilleras Béticas $=\mathrm{CB})$ y colinda al S con el Mar Mediterráneo; tiene diferencias altitudinales, como las montañas Béticas y su Depresión adyacente, en esta última hay otras estructuras (el Surco Intrabético y el Valle de los Pedroches). Andalucía tiene importantes sistemas de montaña, pero las llanuras son $\sim 40 \%$ (altitud media $\sim 500 \mathrm{~m}$ ). La costa posee muchos y variados contrastes morfológicos, hipsométricos y tipológicos (playas y costas bajas) (Figura 3A). Hacia $\mathrm{M}$ se incrementan los acantilados y cañones fluviales (Figura 3B).

Las CB son el principal y mayor bloque del S de la PI. Hay varias Sierras (Campiña, Córdoba, Huelva, Jaén, Morena (Pico Estrella =1,300 $\mathrm{m})$, Sevilla y Ubrique $(1,654 \mathrm{~m})$ ). Aquí está el Sistema Penibético, una barrera morfológica continua entre el litoral y el interior peninsular. Los ríos han configurado áreas, relativamente, aisladas (Alpujarras, Altiplanicie de Vélez, Cazorla (>1,000 m), Desierto de Tabernas, Montes de M, Serranía de Ronda (Pico de las Nieves =1,919 m)-Grazalema, Tablón (Pico Terril $=1,130 \mathrm{~m}$ ) y Tierra de Alhama). Esta somera y general descripción del relieve muestra la diferenciación morfoestructural, y es el primer paso en la clasificación morfotectónica de C-M y la determinación de sus sectores más activos.

\section{Principales características geológicas y tectónicas}

En la clasificación morfotectónica se han utilizado, principalmente, los estudios geológicos de: Calvert et al. (2000), Capote et al. (2002), Galindo-Zaldívar et al. (1993), Negredo et al. (2002), Reicherter y Peters (2005) y Soto y Manzano (2002). Ellos permiten asegurar que Andalucía: a) tiene un desarrollo geológico desde el Precambrico ( 470 Ma); b) los materiales más antiguos están en la Zona Ossa-Morena; c) sufrió plegamientos (huroniano, caledoniano, y hercinico) que modelaron la estructura al SW-NE y tuvo un largo periodo de estabilidad tectónica; d) en el Mioceno Superior-Mioceno Inferior sufrió la compresión NW-SE de la orogenia 
Alpina, derivando a N-S y NNW-SSE; e) hay fallas con esas direcciones; f) surge y evoluciona hasta el presente por la interacción de las placas África y Euroasiatica; g) se localiza en el Macizo Hercínico de La Meseta y la parte septentrional de las $\mathrm{CB}$; h) las montañas meridionales de la $\mathrm{CB}$ han tenido un levantamiento general desde el Plioceno Inferior, lo que ha configurado la actual línea de costa del Mar de Alborán.

Destacamos que: 1) el movimiento relativo (1-2 mm/año) entre las placas Eurasiática y Norteamérica desde hace $3 \mathrm{Ma}$; 2) al SW de la PI (Faro, cerca del Cabo de San Vicente, - las islas Azores) el relieve es netamente submarino con contrastes batimétricos y de pendientes. Hay varias morfoestructuras (Banco de Gorringe y 2 llanuras de tipo abisal Horseshoe y Tagus); 3) el sector Faro-C contiene el cambio de tipo de corteza (oceánica-continental) y donde los contrastes son entre estructuras submarinas (Golfo de C y Mar de Alborán) con el megabloque PI y el N de África); 4) al W de Azores-Gibraltar hay un complejo patrón de deformación compresiva desde el Mesozoico; 5) el relieve submarino de Marruecos-PI, entorno al Estrecho de Gibraltar, tiene diferentes morfoestructuras (Umbral de Camarinal, el Monte Hércules, Cuenca de Tarifa, Cresta Hispali, Monte Tartesos, Hoyas de Poniente y Hoyas de Levante); 6) en Azores-Estrecho de Gibraltar se distinguen 3 segmentos tectónicos.

Vemos que: 1) la modelización de la zona de contacto neotectónico África-América, desde la Cordillera Medio Oceánica a Argelia, tiene diferentes estilos y tipos de litosfera; las deformaciones están en una estrecha banda. El modelo está mejor ajustado para el segmento Azores-Estrecho de Gibraltar que al E del Estrecho (hasta Argelia); 2) existen para el límite de África-PI-Mar de Alborán 6 zonas de estructuras lineales; 3 ) en AzoresTurquía se han estimado los valores del Tensor del Momento. Se destacan 3 segmentos: a) Azores-Estrecho de Gibraltar tiene 3-12 mm/año; b) Gibraltar-Argelia con $5 \mathrm{~mm} / \mathrm{año}$; c) el extremo oriental del Mediterráneo de hasta $21 \mathrm{~mm} / \mathrm{año}$ ); 4) estudios de la corteza muestran que en la zona costera atlántica hay velocidades en el manto superior de $8.15 \mathrm{~km} / \mathrm{s}$ con profundidades de $30 \mathrm{~km}$, y en las inmediaciones del Algarve (S de Portugal) la profundidad alcanza $35 \mathrm{~km}$; 5) la región Bética-Rifeña tiene modelos geodinámicos, entre ellos destacamos 3: a) año 1984 que involucra a 4 fallas transcurrentes de distinta edad; b) año 1992 considera que la actual zona de convergencia PI-África tiene una deformación distribuida en el

172 Revista Geográfica de América Central No 55 ISSN 1011-484X, julio-diciembre 2015, pp. 169-198 
marco de fallas transcurrentes; c) año 2002 muestra estilos diferentes de deformación en 2 regiones: c.1) Argelia (la zona más activa del contexto Ibero-Magrebí) con el mayor desplazamiento lateral y sobrecorrimiento; c.2) C-Alicante-Nekor con zonas de fallas de movimiento lateral y normal); 6) de los modelos que explican la subducción de África bajo la PI, tomamos 3 (años 1991, 1993 y 2000), los 2 primeros la asumen para el intervalo Cretácico Superior-Mioceno Inferior y el último para el Neógeno Inferior-Cuaternario.

\section{Algunos datos sobre la sismicidad}

Hay abundante información sobre la sismicidad del S de la PI (Figuras 1A-B). Aquí empleamos: Buforn et al. (2004, 1995), Gurría y Mezcua (2000), Henares Romero y López Casado (2001), Herraiz et al. (2000), IAG (2002), Martínez (2003), Mezcua y Rueda (2006, 2004), Mezcua et al. (2004) y Sanz de Galdeano et al. (1995). Sostenemos que: 1) la AS se localiza a lo largo del sistema de fallas Azores-Gibraltar-Golfo de C-Mar de Alborán-costa $\mathrm{N}$ de África-Argelia; esto se relaciona con la interacción de las placas de África y Euroasiática; 2) los terremotos más fuertes son 2 (1775.11.01 (I=10 MSK, M $~ 8.5$ y 15,000 muertos; SW del Cabo de San Vicente) y 1884 (I=9-10 MSK, M=6.5 y 839 muertos; Arenas del Rey, Granada)); 3) el SW de Portugal-Golfo de C-Marruecos tiene un alto peligro por la ocurrencia de terremotos fuertes y tsunamis; 4) el territorio de C-M ha sufrido 5 importantes terremotos $(1494.01 .26$, I= 8; 1581.06.18, $\mathrm{I}=7-8 ; 1680.10 .09, \mathrm{I}=8-9 ; 1767.07 .17, \mathrm{I}=7 ; 1722.12 .27, \mathrm{I}=8$. Todas las intensidades en escala MSK); 5) la magnitud máxima estimada es $6.8 ; 6$ ) se distinguen 2 rangos de profundidad de los terremotos $(<40 \mathrm{~km}$ y hasta $150 \mathrm{~km}$ ); 7) los mecanismos de muchos terremotos muestran compresiones NNW-SSE, aunque hay E-W; 8) el entorno de Vélez M-Torrox-Verja (S de Granada) ha producido al menos 4 terremotos profundos $(\mathrm{h}=637-660$ $\mathrm{km}$ ), con magnitudes de 4.4-7.0 y los mecanismos focales asociados son muy similares entre sí y difieren de los determinados para las profundidades menores; 9) la simicidad histórica tiene valores muy superiores en magnitud estimada e intensidad que para el periodo instrumental; 10) en este último periodo, con la red de estaciones sísmicas, se tienen muchos eventos de magnitudes bajas; 11) para las coordenadas $-11.00 \mathrm{~W}-3.50 \mathrm{E}$ y $35.00 \mathrm{~N}-39.00 \mathrm{~N}$ se han determinado, a partir de los parámetros $\mathrm{M}_{0}$, 
velocidad de deslizamiento, y el parámetro b, 3 zonas sísmicas; 12) han sido delimitadas 23 fuentes sísmicas en las CB-Rif-parte del Tell y, se subdividen en 4 Grupos y varios Subgrupos. Las de nuestro interés son de 3 Grupos: Grupo I (con 4 tramos: Carmona-Córdoba-Jaén, Golfo de C, Ubrique-Antequera y M-Adra); Grupo II (con 3 tramos: Alhama de Granada, Granada y Almería); Grupo III (Alhucemas-Isla de Alborán); 13) para C hay 5 isosistas de terremotos: a) 13.03.2002 en Prado del Rey (Imáx $=4$ y figura circular); b) 30.03.2002 en San José del Valle (Imáx $=3$ y figura elíptica con eje principal E-W); c) 25.07.2003 en Puerto Serrano (Imáx=4 y figura elíptica con eje principal NW); d) 05.02.2009 en Coripe (Imáx = 3 y figura circular); e) 21.06.2010 en Jerez de la Frontera (Imáx= 4 y figura elíptica con eje principal NE)). Pero en $M$ sólo hay medias isosistas, ya que los epicentros están en su inmensa mayoría en el mar, como por ejemplo: el terremoto del 21.11.2002 (M=4,2 / Imáx=4) frente a la ciudad de Málaga); 14) para el segmento de falla entre las localidades de M y Adra se asegura hay relación con los terremotos y el levantamiento de la costa.

174 Revista Geográfica de América Central No 55 ISSN 1011-484X, julio-diciembre 2015, pp. 169-198 
Figura 1. Localización del segmento Cádiz-Málaga en la Península Ibérica
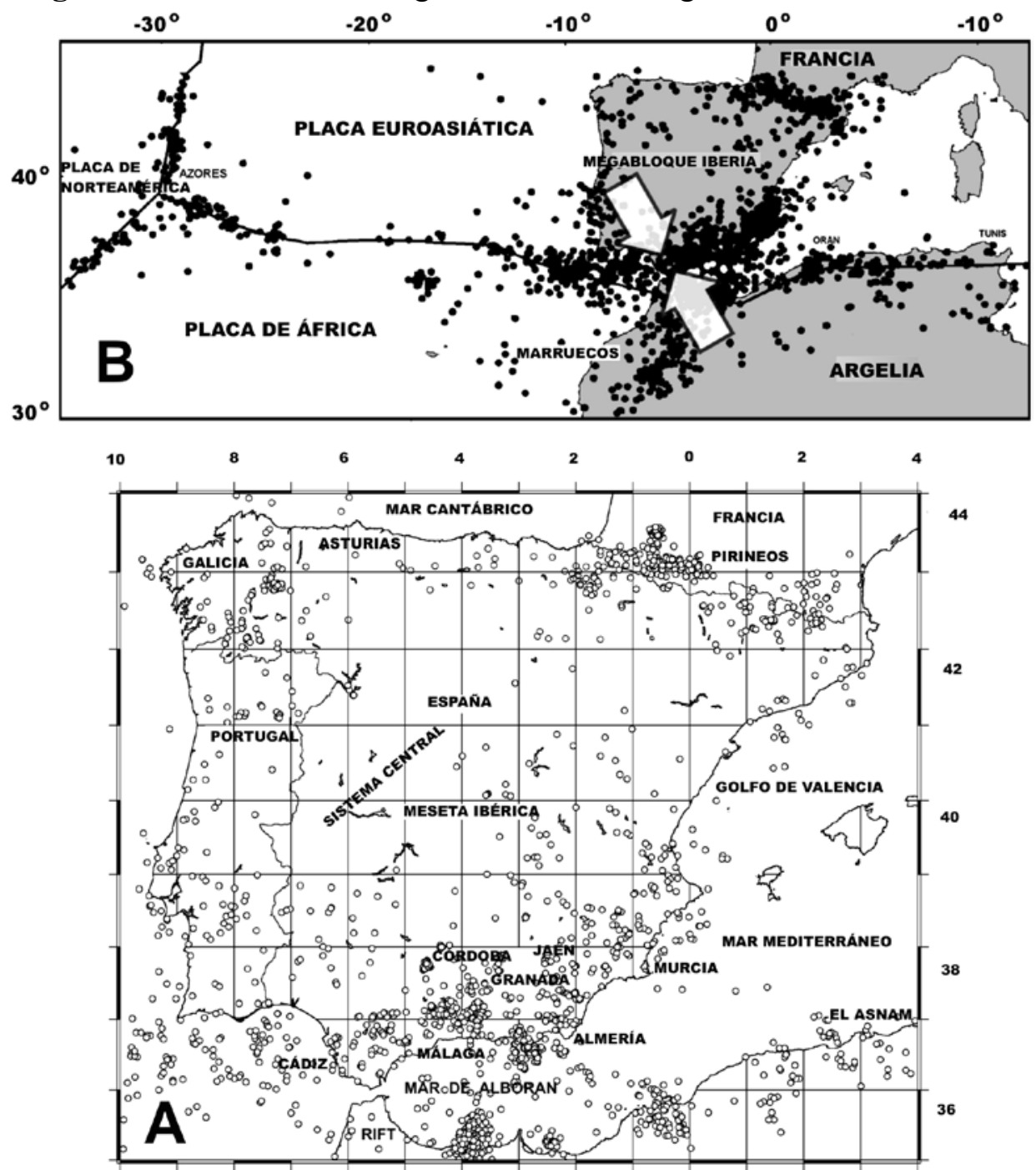

A) Selección de epicentros de terremotos en la Península Ibérica. (Están indicados: 1) epicentros (círculos); 2) regiones de los estudios morfotectónicos realizados (Córdoba-Granada, AlmeríaJaén, Asturias, Galicia, Murcia, Península Ibérica y Sistema Central)).

B) Zona de colisión de las placas África y Euroasiática. (Aparecen: línea de fallas= línea gruesa negra; epicentros $=$ puntos negros; flechas gruesas blanca $=$ sentido de la convergencia). 
Mario Octavio Cotilla-Rodríguez, Diego Córdoba-Barba. Morphotectonics of the Cadiz-Malaga Segment, Southern Spain

Figura 2. Localización del segmento Cádiz-Málaga en Andalucía
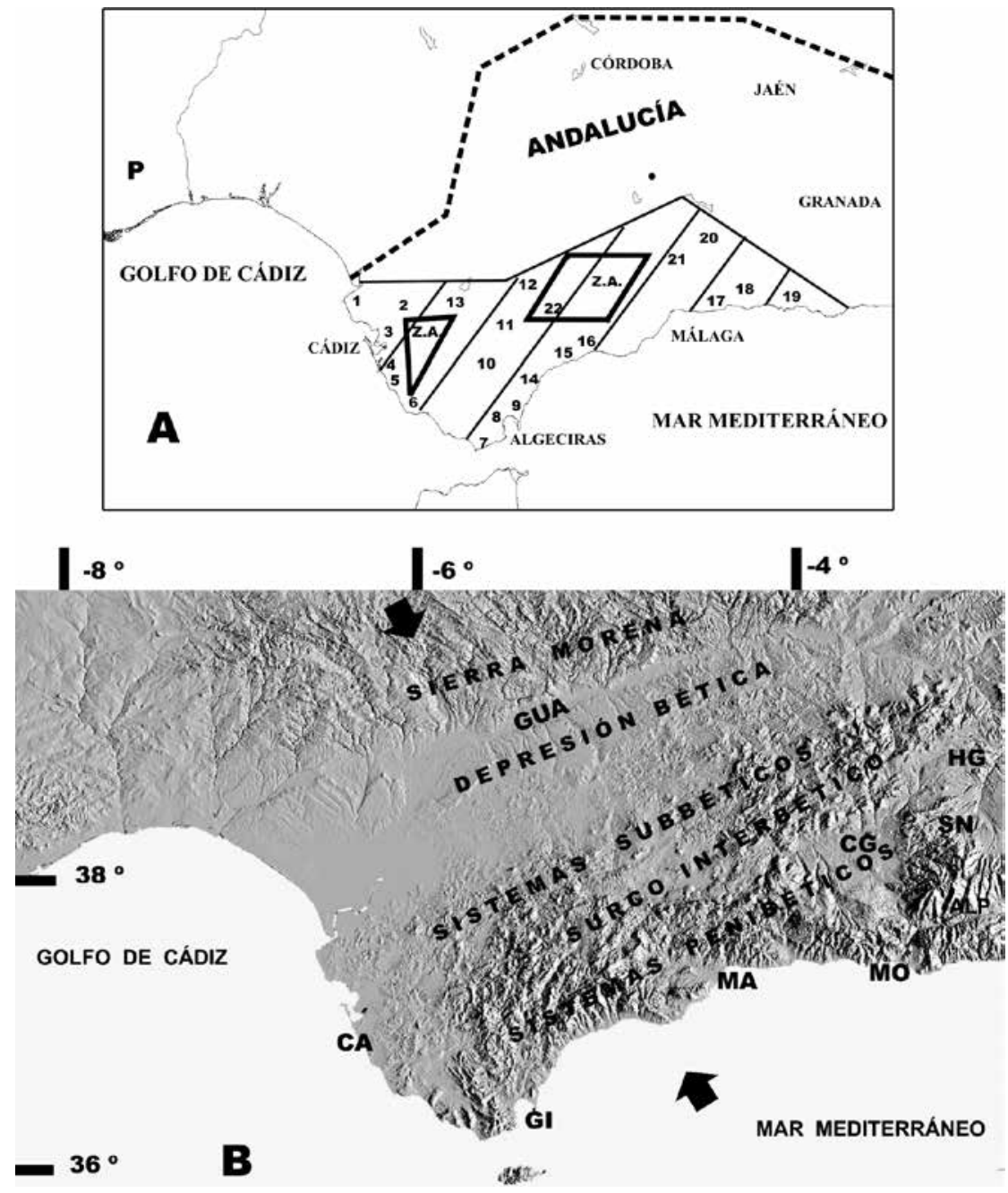

A) Provincias de Cádiz y Málaga. (Se enmarcan la mayor parte de la Comunidad de Andalucía y las zonas de estudio Cádiz y Málaga, que están indicadas con líneas transversales. Algunas localidades de interés aparecen con números: 1= Sanlúcar de Barrameda, 2= Jerez de la Frontera, 3= El Puerto de Santa María, 4= San Fernando, 5= Chiclana de la Frontera, 6= Barbate, 7= Tarifa, 8=Algeciras, 9= La Línea de la Concepción, $10=$ Alcalá de los Gazules, $11=$ Ubrique, 12= Puerto Serrano, 13=Arcos de la Frontera, 14= Estepona, 15= Marbella, 16= Fuengirola, 17= Rincón de la Victoria, 18=Vélez-Málaga, 19= Nerja, 20=Archidona, 21=Antequera, 22= Ronda. Hay un triángulo y un paralelogramo que son zonas activas (Z.A.)).

B) Principales sistemas del relieve en la región de estudio. (Aparecen: 1) localidades: $\mathrm{CA}=\mathrm{Cádiz}$, $\mathrm{MA}=$ Málaga, $\mathrm{MO}=$ Motril, $\mathrm{GI}=$ Gibraltar; 2) flechas gruesas negras= sentido de la convergencia de las placas; 3) zonas de interés: $\mathrm{ALP}=$ Alpujarras, $\mathrm{CG}=$ Cuenca de Granada, $\mathrm{GUA}=$ cuenca del Guadalquivir, $\mathrm{HG}=$ Hoya de Granada y $\mathrm{SN}=$ Sierra Nevada).

176 Revista Geográfica de América Central No 55 ISSN 1011-484X, julio-diciembre 2015, pp. 169-198 


\section{Evaluación morfotectónica}

\section{Características morfotectónicas}

La configuración, la disposición y los valores de las morfoisohipsas muestran un relieve segmentado en familias de bloques desde un tope máximo de $\sim 2,000$ de altitud. Las cotas altimétricas son inferiores en C con respecto a M. Hay diferencias en la orientación de los ejes principales de los bloques delimitados, predominando las direcciones N-S y $\mathrm{N}$ en $\mathrm{C}$ y $\mathrm{NE}$, E-W y N-S en $\mathrm{M}$. El relieve en $\mathrm{M}$ tiene un significativo escalonamiento de dirección SE-NO, al menos en 5 niveles. También, M muestra una diferenciación hipsométrica en 2 niveles, con isohipsas paralelas a la costa de: 1) $500 \mathrm{~m}$ [Coeficiente de Sinuosidad= Ks] $(\mathrm{Ks}=0.63)$; 2) 1,000 m $(\mathrm{Ks}=0.36)$; los valores de pendientes medias son muy superiores con relación a $\mathrm{C}$.

Figura 3. Fotos de la región de trabajo

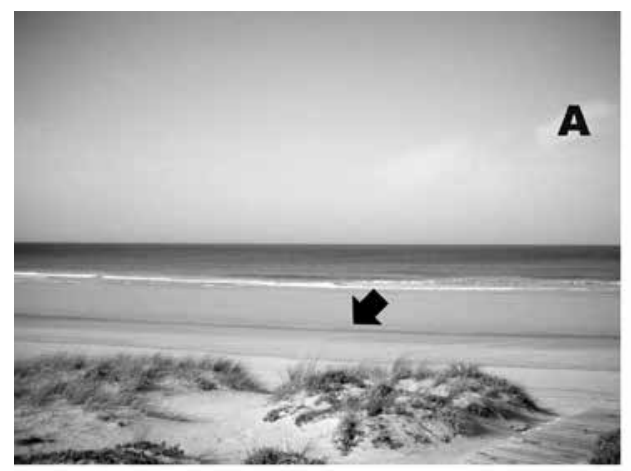

A) Playa en Cádiz (Se indican algunas pequeñas dunas (flecha negra gruesa)).

B) Acantilado en Málaga (Se aprecian el corte vertical de la costa, los fragmentos de bloques desprendidos (flechas gruesas negras) y la estructura geológica).

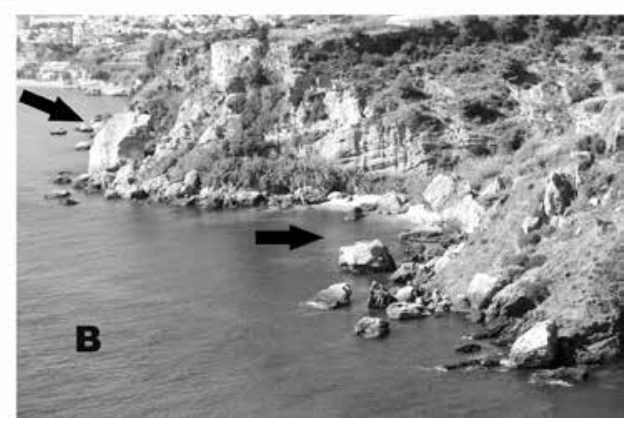


Mario Octavio Cotilla-Rodriguez, Diego Córdoba-Barba. Morphotectonics of the Cadiz-Malaga Segment, Southern Spain

La combinación supervisada de las morfoisohipsas, la red fluvial (Figura 4) y las fotos, ha permitido determinar 30 morfoalineamientos; 22 en C y 8 en $M$ (Figura 5). En C existe un sistema de 4 alineaciones (NW) que configura un escalonamiento morfoestructural. Ellos se visualizan, también, con las Divisorias Principales o Parteaguas Principales [PP] de $1^{\text {er }}$ y $2^{\circ}$ orden que limitan todo el $\mathrm{N}$ de las Sierras Costeras. De las 8 alineaciones delimitadas, en $\mathrm{M}$ hay 4 relacionadas con la costa. Entre $\mathrm{C}$ y $\mathrm{M}$ aparece el alineamiento NE (LB1). Este sistema de fracturas es ligeramente diferente al determinado en $\mathrm{C}$, ya que las alineaciones $\mathrm{E}-\mathrm{W}$ son mucho más numerosas; es decir, existe una red de importantes fracturas que permiten asumir los efectos en el relieve de la actual convergencia de las placas.

Figura 4. Esquema de la red fluvial superficial de Andalucía

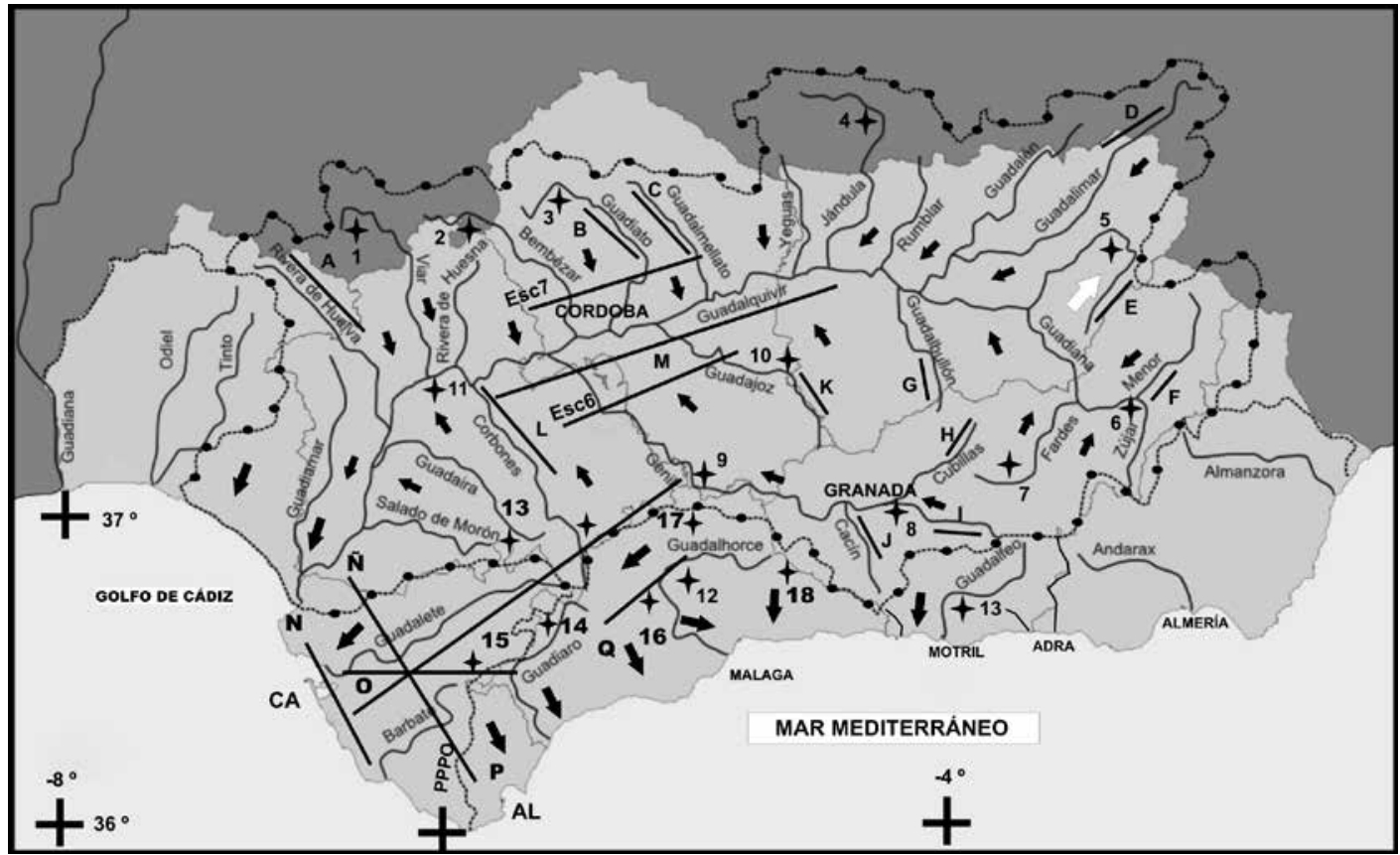

Están representados: 1) los ríos principales; 2) las principales alineaciones fluviales (líneas negras gruesas, con una letra (A)); 3) el parteaguas principal de primer orden (línea negra con puntos); 4) algunas zonas con anomalías fluviales (estrella negra, con un número); 5) el sentido principal del escurrimiento superficial (flechas gruesas en negro). 
La costa de $\mathrm{C}$ se subdivide en 8 tramos morfológicos (Figura 5): 1) Punta del Perro (Chipirona)-Rota; 2) Rota-C; 3) C-Cabo Roche; 4) Cabo Roche-Cabo Trafalgar; 5) Cabo Trafalgar-Punta Camarinal; 6) Punta Camarinal-Punta de Tarifa; 7) Punta de Tarifa-Punta Carnero y 8) Punta de Europa-Punta Chullera ( $\mathrm{M}$ está al E). Esos segmentos se distinguen por la dirección y el Ks. Así, para los tramos: a) 1-4 predomina la dirección NW y hay un $\mathrm{Ks}=0.98$; b) 5-6 también de dirección NW, pero diferentes Ks (tramo $5=0.88$ y tramo $6=0.75$ ); c) 7 es de dirección NE y Ks=0.95; d) 8 con dirección $\mathrm{NNE}$ y $\mathrm{Ks}=0.90$. Mientras que la costa en $\mathrm{M}$ tiene un Ks= 0.72; también, se define sobre la base de la longitud, la dirección principal y el Ks. Así tenemos de O-E: 1) Punta de la Chullera-Estepona (16 km / NNE / 0.96); 2) Estepona-Ensenada de Marbella (20 km / NE / 0.91); 3) Ensenada de Marbella-Ensenada de Cala Moral (22 km / E-W / 0.95); 4) Ensenada de Cala Moral-Punta Negra-Torremolinos-Ensenada de M (30 $\mathrm{km} / \mathrm{NNE} / \mathrm{0.90)}$; 5) Ensenada de M-Punta de Vélez-M-Torrox-CostaNerja (56 km / E-W / 0.93).

La disposición e hipsometría de la costa y su forma son muy diferentes para $\mathrm{C}$ y $\mathrm{M}$; en este sentido, el Ks promedio, es también diferente en $\mathrm{C}$ (0.48) y M (0.65). Resalta la figura cóncava al S de la Bahía de Algeciras en $\mathrm{C}$ como una característica peculiar morfológica del territorio, esto se ajusta bastante bien, a sistemas de las formas del relieve triangular irregular en $\mathrm{C}$ y otro rectangular para $\mathrm{M}$. 
Figura 5. Esquema de alineaciones y epicentros

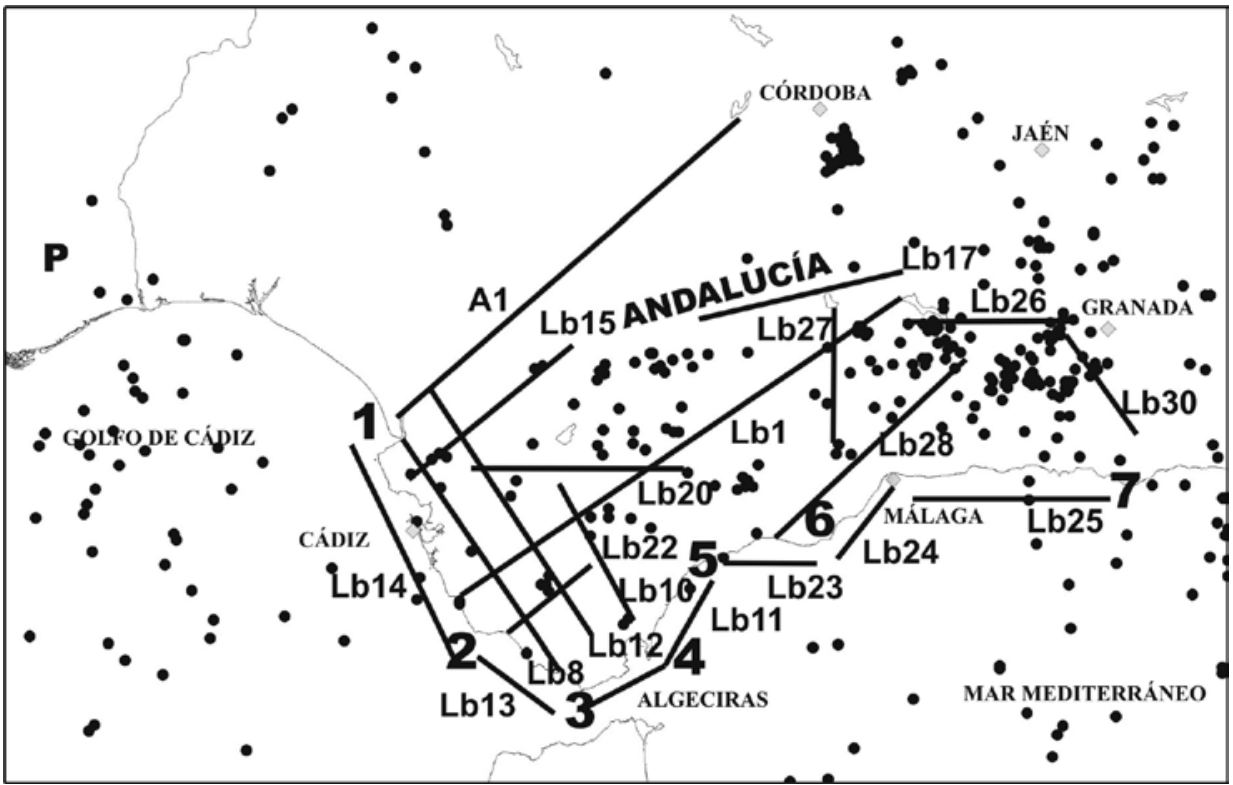

Aparece: 1) Selección de epicentros (círculos negros); 2) alineaciones principales (A1, Lb2) (Ver Tablas 7 y 8); 3) tramos de costa (1-7).

La provincia de $\mathrm{C}$ está al $\mathrm{S}$ del río Guadalquivir (Figura 4). Su hipsometría tiene un débil escalonamiento, así se localizan valores $>500 \mathrm{~m}$ en la parte NE de la provincia. Las Sierras Costeras están localizadas al E de Barbate y del Cabo de Trafalgar; mientras que el resto de las Sierras está dispuesto en 2 escalones morfoestructurales N-S.

En $\mathrm{C}$ y $\mathrm{M}$ hay $\sim 1,000$ corrientes fluviales y una variada diferenciación por cuencas. La categoría, disposición y geometría de las cuencas es diferente; así es significativo el número de ríos con anomalías; para $\mathrm{C}$ (98) y M (267). Las características de los valles principales para esas regiones se diferencian entre sí, aunque se distingue un patrón regional. El desarrollo e instalación de la red fluvial en C y M están definidos desde el Plioceno. En los diseños están tanto la jerarquía de los ríos como la configuración de las cuencas, divisorias de agua y terrazas fluviales. Las mayores cuencas fluviales de: 1) C son de los ríos Guadalete (NE-SW), Barbate $(\mathrm{N}-\mathrm{S})$ y Guadiaro (NE). El gradiente de los ríos difiere (Guadalete $=0.4$ $\mathrm{m} / \mathrm{km}$, Barbate $=0.8 \mathrm{~m} / \mathrm{km}$ y Guadiaro $=1.3 \mathrm{~m} / \mathrm{km}$ ). Sus desembocaduras 
están en la Bahía de Cádiz (Guadalete), cerca del Cabo de Trafalgar (Barbate) y el Guadiaro que corta a la Sierra Carbonera para llegar al NE de La Línea de la Concepción. Los Ks tienen valores de 0.55 (Barbate) y 0.81 (Guadiaro); 2) En M son los ríos Guadalhorce (NW) y Benamargos (NW). El río Guadalhorce ( $6^{\circ}$ orden) desemboca entre Torremolinos y M; y el Benamargos ( $5^{\circ}$ orden) lo hace en Vélez-M, con un gradiente de 1.6 y 1.9 $\mathrm{m} / \mathrm{km}$, respectivamente. El drenaje en $\mathrm{M}$ se distingue por la dirección: 2.1) NW-SE (en Punta de la Chullera-Maro y en Barbate-Punta de la Chullera); 2.2) NE-SW (C-Rota-Barbate).

El entorno del Golfo de C (localidades de: Beja-Sevilla-C-Villa Real de Santo Antonio-Faro) tiene terrazas fluviales del NE-SW (ríos Guadalquivir, Odeil y Tinto); las más jóvenes con dirección N (Gualdaquivir-Tinto-Odeil-Guadiana). En las inmediaciones de Beja hay terrazas (E-W de niveles superiores asociadas al río Guadiana. Esto se corresponde con las inflexiones y las alineaciones de los ríos tributarios y las cuencas (OdeilTinto y Guadalquivir). Indicamos que el Guadalquivir y el Guadalete comparten la misma área de cotas bajas y planas (El Puerto de SantamaríaLebrija-Las Cabezas de San Juan), donde están las principales alineaciones, mencionadas anteriormente.

Datos de la orientación del drenaje superficial indican que tiene dirección predominante N-S, aunque los del NE son altos. Las anomalías fluviales son mayores en la zona meridional de $\mathrm{C}$ con relación a M; pero, el porciento de las Relaciones de Bifurcación anómalas resulta ser inferior para los ríos de menor orden de $\mathrm{C}$ que para los de $\mathrm{M}$; esto se interpreta como una mayor actividad de tipo vertical en la región de $\mathrm{M}$.

Los PP delimitados en C son de 4 órdenes: Primero (vertiente $\mathrm{N}$ de los 2 ríos de $6^{\circ}$ orden); Segundo ( 3 al S y 3 al N); Tercero ( 2 al S y 7 al N) y Cuarto (4 al N). El dibujo hipsométrico en el territorio de C-M es muy complejo e irregular. Existe basculamiento de las estructuras en C: 1) al $\mathrm{N}$ del PP de $1^{\text {er }}$ orden del NE-SW; 2) al S del PP de $1^{\text {er }}$ orden del NO-SE. Pero no lo hemos determinado en M. Hay diferencias en el total de escalones morfoestructurales del relieve, $\mathrm{C}$ y $\mathrm{M}$ tienen 4 y 7, respectivamente. La intensidad de los levantamientos neotectónicos muestra sectores de distinto orden y sus valores representan el $33 \%$ en C y el $82 \%$ en M. Además, la cantidad de superficies de cimas delimitadas difiere en ambos territorios, esto permite inferir la diferencia en actividad de tipo vertical, siendo mucho mayor en $\mathrm{M}$ que en $\mathrm{C}$. 
En M las cuencas de $4^{\circ}$ orden, delimitadas por el PP de ese mismo orden, tienen ciertas características de interés tectónico. Así, en M-Marbella hay una extensa cuenca que es paralela a la línea de costa, dirección E-W. Mientras que al E de M (Marbella-Punta de la Chullera) son 8 las cuencas de $4^{\circ}$ orden (mucho menores en área que la localizada en la parte W), y tienen dirección NW-SE. En las cuencas de $5^{\circ}$ y $6^{\circ}$ orden (M-Vélez M) se identifica otra serie de elementos del relieve paralelos a la costa. Así, es factible asumir que la diferenciación, con respecto a $\mathrm{C}$, está justificada por variaciones laterales en la convergencia de las placas.

Hay 7 alineaciones de $\mathrm{C}$ que se relacionan con inflexiones de los PP: 1) Jerez de la Frontera-Medina-Sidenia-Benalup-Casas Viejas (dirección NW, 64 km); 2) San Fernando-Vejer de la Frontera-Barbate (NW, paralelo al anterior, $40 \mathrm{~km}$ ); 3) Algeciras-Los Barrios-Embalse del Guadalcin II (dirección NNW, 46 km); 4) Sierra del Aljibe-Sierra de Libar-Sierra del Palo-Ronda (M) (dirección NE, 38 km); 5) La Barca de la Honda-Arcos de la Frontera-Villamartín-Puerto Serrano (dirección NE, 38 km); 6) SevillaMontellano-Sierra del Pozo Amargo (dirección E-W, 38 km); 7) Rota-El Cuervo ( $\mathrm{N}$ de Jerez de la Frontera) (dirección NE, $46 \mathrm{~km}$ ). Estas alineaciones se corresponden con segmentos de las 30 alineaciones determinadas con las morfoisohipsas y las fotos.

El río Guadiaro, en el extremo NW de M, tiene dirección NNE-SSW y corre paralelo al PP $1^{\text {er }}$ orden (al W de Roda) entre las Sierras de las Salinas, del Palo, Blanquilla y de los Prios. El río va encajado entre Jimena de Libar y las Sierras del Palo y Blanquilla, con un $\mathrm{Ks}=0.98$. Determinamos 3 anomalías y asimetría transversal en la parte SE (en Sierra de los Pinos y Cortes de la Frontera). Paralelo y al SE de este río aparece el río Genal que discurre encajado por la Serranía de Ronda. En él hemos detectado varias afectaciones por fracturas transversales y perpendiculares en Faraján-Benalauria-Benarrabá-Gaucín que reducen el valor del Ks a 0.78. Esta zona está al NW de las de cuencas de $4^{\circ}$ orden que drenan entre Estepona y Marbella, ya comentadas; este entorno fluvial y sus deformaciones son resultado de compresiones NE. 
Figura 6. Unidades Territoriales mayores

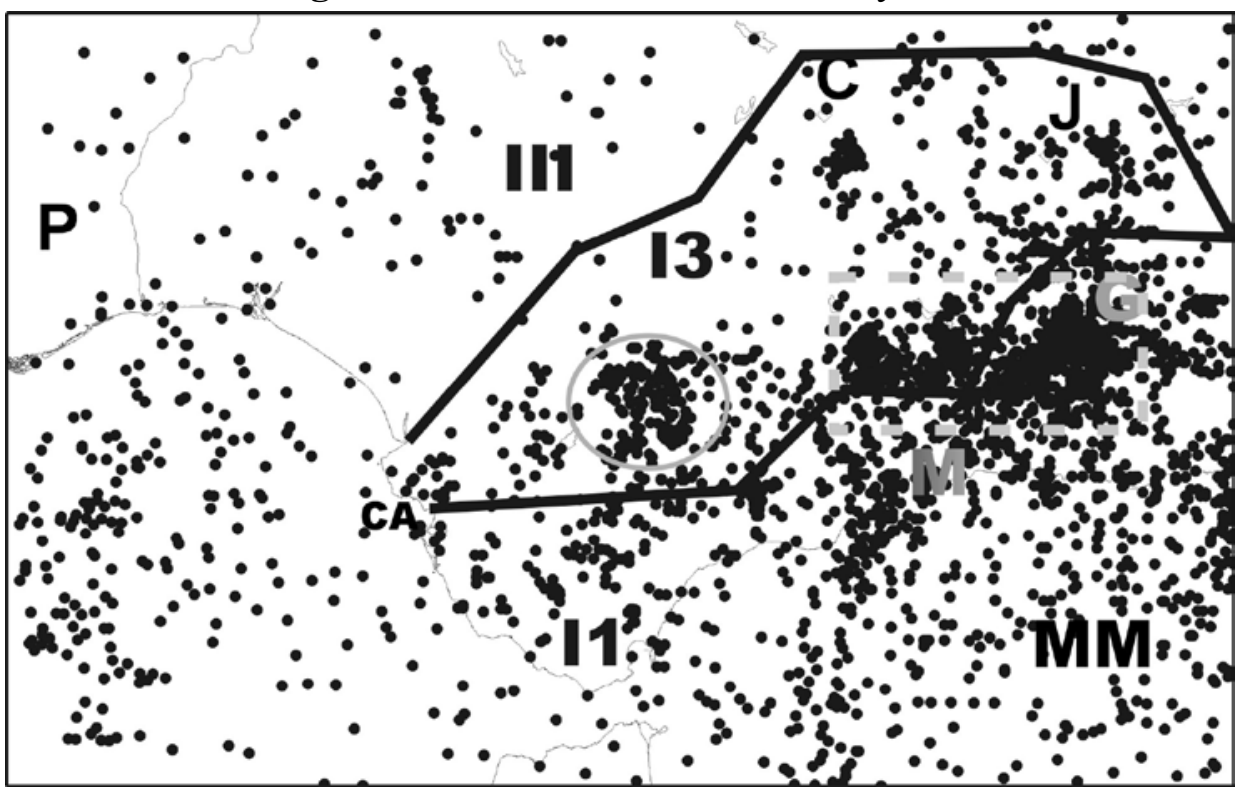

Aparecen: 1) los macrobloques (I y II); 2) mesobloques (I1 y I3); 3) localidades (C= Córdoba, CA= Cádiz, $\mathrm{G}=$ Granada, $\mathrm{J}=$ Jaén, $\mathrm{M}=$ Málaga, MM= Mar Mediterráneo); 4) epicentros de terremotos= círculos negros; 5) círculo y rectángulo señalan áreas de agrupaciones epicentrales.

Del estudio de la PI (Cotilla y Córdoba, 2004) hay en Andalucía 5 alineaciones principales de $1^{\text {er }}$ y $2^{\circ}$ orden (A1, A2, L1, L2, y L3) y 6 nudos de $1^{\circ}-3^{\text {er }}$ orden $(\mathrm{N} 1, \mathrm{~N} 2, \mathrm{~N} 3, \mathrm{~N} 6$, y N7); sin embargo, en C y M solo están 3 alineaciones A1, A2 y L1, pero los nudos no. En las inmediaciones de Motril (Almería) está el nudo N1, que dista, aproximadamente, $20 \mathrm{~km}$ de la región de $\mathrm{M}$; por consiguiente, tiene influencia en ella.

Datos del macrobloque ( $\mathrm{I}=$ Merdional) en C y M están en la tabla 1. En las tablas 2-5 y la figura 6 aparecen las UT de menor orden. Los 2 territorios están en los mismos mesobloques del S de la PI (denominados I1 y I3). El I1 (6/8 partes del total) tiene el mayor área y es el más activo. La zona de M (Figura 7) es más activa que la de C. En la figura 6, hay 2 agrupaciones epicentrales (indicadas con un círculo y un rectángulo), que luego del procesado con diferentes filtros delimitan 2 zonas sismoactivas (Figura 2A). 
Mario Octavio Cotilla-Rodríguez, Diego Córdoba-Barba. Morphotectonics of the

Cadiz-Malaga Segment, Southern Spain

Tabla 1. Características de los macrobloques

\begin{tabular}{|c|c|c|}
\hline Características & Cádiz & Málaga \\
\hline Área $\left(\mathrm{km}^{2}\right)$ & $\sim 3,300$ & $\sim 4,000$ \\
\hline Longitud de costas $(\mathrm{km})$ & 1,620 & 1,490 \\
\hline Altitud máxima (m) & $\sim 1,900$ & $\sim 1,900$ \\
\hline Altitud media (m) & $\sim 490$ & $\sim 700$ \\
\hline Cantidad de Divisoria Principal de Primer Orden & 1 & 1 \\
\hline Dirección predominante de la DPPO & NE-SW & $\mathrm{N}-\mathrm{S}$ \\
\hline Coeficiente de sinuosidad de la DPPO & 0.70 & 0.56 \\
\hline Cantidad de cuencas fluviales (Septentrionales / Meridionales) & $5 / 240$ & $20 / 565$ \\
\hline Orden máximo de los ríos & 6 & 6 \\
\hline Cantidad de sectores anómalos en los ríos & 98 & 267 \\
\hline Cantidad de superficie de cimas & 158 & 464 \\
\hline Cantidad de mesobloques / bloques / microbloques / nanobloques & $2 / 18 / 98 / 192$ & $2 / 11 / 136 / 206$ \\
\hline Cantidad de alineaciones principales & 17 & 12 \\
\hline Cantidad de intersecciones principales $3^{\text {er }} / 4^{\circ}$ orden & 234 & 398 \\
\hline Cantidad de nudos $\left(3^{\mathrm{er}} / 4^{\circ}\right.$ orden $)$ & $8 / 12$ & $5 / 6$ \\
\hline Dirección predominante de las fracturas & N-S & N-S \\
\hline Magnitud del terremoto más fuerte & 6.5 & 6.8 \\
\hline
\end{tabular}

Nota: $\mathrm{DPPO}=$ Divisoria Principal de Primer Orden.

Tabla 2. Composición de los mesobloques

\begin{tabular}{|l|c|c|c|c|c|}
\hline Provincia & Bloques & Microbloques & Nanobloques & $\begin{array}{c}\text { Intersecciones// } \\
\text { nudos }\end{array}$ & $\begin{array}{c}\text { Valles } \\
\text { (V/U) }\end{array}$ \\
\hline Cádiz & 18 & 98 & 192 & $265 / 20$ & $63 / 95$ \\
Málaga & 11 & 136 & 206 & $481 / 12$ & $142 / 161$ \\
\hline Total & 29 & 234 & 398 & $746 / 32$ & $205 / 256$ \\
\hline
\end{tabular}

Las principales características de los sistemas de alineaciones se presentan en las tablas 6-8. Las intersecciones o nudos principales entre estos elementos se dan las tablas 6 y 9. La información permite valorar las zonas y áreas más activas. Ellas se localizan tanto en la zona de costa, como en el interior del territorio, siendo relevantes aquellos que son los más extensos, como por ejemplo el L1 en $\mathrm{C}$ y $\mathrm{M}$. El total de nudos configurados con las alineaciones principales resulta diferente para $\mathrm{C}=20$ y $\mathrm{M}=12$. Esto se explica a partir de las longitudes de las alineaciones y

184 Revista Geográfica de América Central No 55 ISSN 1011-484X, julio-diciembre 2015, pp. 169-198 
su regularidad espacial. Los morfoalineamientos coinciden en sectores o segmentos con los presentados a partir de los estudios de morfoisohipsas y de la red fluvial.

Los valores de la fracturación del relieve y las características de las fracturas por bloques en $\mathrm{C}$ y $\mathrm{M}$, resultan ser similares. Así hay en $\mathrm{C} \mathrm{y} \mathrm{M}$, 0.03 y 0.04 (cantidad de fracturas $/ \mathrm{km}^{2}$ ), respectivamente. Relacionado con esto está la afectación a estructuras de circulación vial, 9 en cada una. También, se han visto algunos corrimientos de laderas, de pequeña magnitud, como en Prado del Rey, inmediato a la carretera de El Bosque; y en el río Guadalete, cerca de Puerto Serrano.

Tabla 3. Datos de los bloques en Cádiz / Málaga

\begin{tabular}{|l|c|c|c|c|c|c|c|}
\hline & & & & & \multicolumn{2}{|c|}{ Valles } & \multicolumn{2}{c|}{ Superficies de cimas } \\
\cline { 5 - 8 } B & $\mathbf{M}$ & $\mathbf{N}$ & Dirección & $\mathbf{V}$ & $\mathbf{U}$ & Planas & Puntiagudas \\
\hline 1 & $2 / 10$ & $4 / 21$ & N-S/NE & $-/ 16$ & $2 / 21$ & $5 / 26$ & $-/ 39$ \\
2 & $4 / 8$ & $10 / 15$ & NW/N-S & $-/ 20$ & $4 / 18$ & $5 / 17$ & $-/ 25$ \\
3 & $4 / 10$ & $11 / 17$ & NW/N-S & $1 / 12$ & $5 / 14$ & $6 / 15$ & $-/ 19$ \\
4 & $6 / 18$ & $15 / 26$ & NW/N-S & $6 / 19$ & $9 / 20$ & $5 / 25$ & $2 / 32$ \\
5 & $5 / 26$ & $11 / 41$ & NW/N-S & $6 / 23$ & $9 / 27$ & $7 / 35$ & $2 / 47$ \\
6 & $5 / 12$ & $11 / 15$ & NW/N-S & $5 / 17$ & $9 / 7$ & $5 / 16$ & $3 / 25$ \\
7 & $4 / 5$ & $7 / 7$ & N-S/NW & $1 / 2$ & $3 / 3$ & $4 / 4$ & $-/ 1$ \\
8 & $3 / 12$ & $5 / 20$ & N-S/N-S & $2 / 16$ & $3 / 20$ & $5 / 25$ & $-/ 30$ \\
9 & $6 / 16$ & $12 / 23$ & N-S/N-S & $4 / 17$ & $6 / 15$ & $7 / 21$ & $1 / 33$ \\
10 & $6 / 9$ & $14 / 11$ & N-S/N-S & $7 / 3$ & $7 / 7$ & $9 / 15$ & $4 / 5$ \\
11 & $8 / 10$ & $16 / 10$ & N-S/N-S & $2 / 7$ & $4 / 9$ & $5 / 14$ & $2 / 5$ \\
12 & $11 /-$ & $19 /-$ & N-S/- & $9 /-$ & $11 /-$ & $9 /-$ & $5 /-$ \\
13 & $14 /-$ & $21 /-$ & NW/- & $14 /-$ & $9 /-$ & $5 /-$ & $7 /-$ \\
14 & $12 /-$ & $20 /-$ & NW/- & $3 /-$ & $2 /-$ & $3 /-$ & $3 /-$ \\
15 & $2 /-$ & $4 /-$ & N-S/- & $-/-$ & $2 /-$ & $5 /-$ & $-/-$ \\
16 & $2 /-$ & $4 /-$ & NE/- & $1 /-$ & $3 /-$ & $4 /-$ & $-/-$ \\
17 & $2 /-$ & $4 /-$ & NE/- & $1 /-$ & $3 /-$ & $4 /-$ & $-/-$ \\
18 & $2 /-$ & $4 /-$ & N-S/- & $1 /-$ & $3 /-$ & $4 /-$ & $-/-$ \\
\hline Total & $98 / 136$ & $192 / 206$ & $-/-$ & $63 / 142$ & $94 / 161$ & $100 / 193$ & $29 / 261$ \\
\hline
\end{tabular}

Nota: $\mathrm{B}=$ bloques, $\mathrm{M}=$ microbloques, $\mathrm{N}=$ nanobloques

\section{Discusión final y conclusiones}

Al emplear más de 40 indicadores, para la clasificación morfotectónica supervisada del relieve, aseguramos que las características del segmento $\mathrm{C}-\mathrm{M}$ tiene diferencias respecto a los 3 segmentos localizados al $\mathrm{E}$ 
Mario Octavio Cotilla-Rodríguez, Diego Córdoba-Barba. Morphotectonics of the

Cadiz-Malaga Segment, Southern Spain

(Almería-Jaén, Córdoba-Granada y Murcia). Todos están en la microplaca Ibérica, y por la metodología aplicada en al megabloque PI. Ellos están en la zona de interacción de placas.

Delimitamos en C-M 5 tipos de UT (macrobloques $=2$, mesobloques $=2$, bloques $=29$, microbloques $=44$ y nanobloques $=202$ ) en relaciones espacio-temporales actuales con 29 alineaciones principales y 32 nudos (Figuras 6 y 7). El 59\% de los bloques es muy activo o activo, y el territorio tiene un valor promedio de $\sim 0.03$ fracturas $/ \mathrm{km}^{2}$.

Tabla 4. Breve descripción de los bloques en Cádiz

\begin{tabular}{|c|l|l|c|}
\hline $\begin{array}{c}\text { Bloques/ } \\
\text { Clasificación }\end{array}$ & \multicolumn{1}{|c|}{ Denominación (localidades) } & \multicolumn{1}{|c|}{ Nudos (Siglas) } & $\begin{array}{c}\text { Mesobloque / } \\
\text { Nudos }\end{array}$ \\
\hline 1/PA & (Puerto Real, Chiclana de la Frontera) & L1, LC11, ALC3 & Mer/5 \\
2/PA & (Paterna de Rivera, Los Islotes) & L1, LC2, LC10 & Mer/5 \\
3/PA & (Sierra Valleja, Sierra de Aznar, Algar) & L1, LC1, LC3 & Mer/4 \\
4/A & (Puerto del Rey, Sierra de Zafalgar) & L1, LC3, LC4 & Mer/3 \\
5/MA & (Algodonales, El Gastor) & L1, LC4, LC5 & Mer/3 \\
6/A & (Alcalá del Valle, Sierra Blanquilla) & L1, LC5 & Mer/2 \\
7/PA & (Medina-Sidaria, Cortijo del Yeso) & LC2, LC7, ALC3 & Mer/3 \\
8/PA & (Conil de la Frontera, Cantabria, Cucarrete) & LC7, ALC2 & Mer/4 \\
9/A & (Sierra Labrada, Sierra de la Sal) & LC1, LC9, LC10, ALC2 & Mer/5 \\
10/MA & (Sierra del Aljibe, Alcalá de los Gazules) & LC1, LC3, LC9, ALC2 & Mer/4 \\
11/A & (Barbate, Sierra de Retín, Algeciras) & LC6, LC8 & Mer/2 \\
12/PA & (Los Alcornocales, Sierra Blanquilla) & LC6, LC8, ALC2 & Mer/3 \\
13/PA & (Sierra de Momia, E1 Jantor, Los Barrios) & LC3, ALC6, ALC2 & Mer/6 \\
14/A & (Zorrera, Cerro Gordo) & LC3, LM6 (Málaga) & Mer/2 \\
15/NA & (Rota, Chipiona, Sanlúcar de Barraeda) & L1, LC12 & Sep/2 \\
16/NA & (Jérez de la Frontera) & L1, LC12, LC13 & Sep/2 \\
17/NA & (Bornos) & L1, LC13, LC14 & Sep/2 \\
18/NA & (Puerto Serrano) & Sep/2 \\
\hline
\end{tabular}

Nota: $\mathrm{A}=$ activo; $\mathrm{MA}=$ muy activo; $\mathrm{NA}=$ no activo; $\mathrm{PA}=$ poco activo; $\mathrm{Mer}=$ meridional; $\mathrm{Sep}=$ septentrional. 
Tabla 5. Breve descripción de los bloques en Málaga

\begin{tabular}{|c|l|l|c|}
\hline $\begin{array}{c}\text { Bloques/ } \\
\text { Clasificación }\end{array}$ & \multicolumn{1}{|c|}{ Denominación (localidades) } & \multicolumn{1}{|c|}{ Nudos (Siglas) } & $\begin{array}{c}\text { Mesobloque / } \\
\text { Nudos }\end{array}$ \\
\hline 1/A & (Gaucín, Loma de los Olivos, Ronda) & L1, LM1, LM6, LM9 & Mer/5 \\
2/A & (Estepona, Sierra Bermeja) & LM1, LM2 & Mer/3 \\
3/A & (Marbella, Coín) & LM2, LM4 & Mer/5 \\
4/A & (Málaga, Camino Real) & LM3, LM7, ALM1 & Mer/2 \\
5/MA & (Vélez-Málaga, Nerja) & L1, LM3 & Mer/3 \\
6/A & (Sierra del Pala, Corte de la Frontera) & LM1, LM5 & Mer/2 \\
7/PA & (Sierra las Cumbres, Cuevas del Becerro) & L1, LM1, LM5 & Mer/2 \\
8/MA & (Alozaina, Sierra Prieta) & L1, LM4, LM6, ALM1 & Sep/4 \\
9/A & (Antequera, Arroyo de los Molinos) & L1, LM4, LM7, ALM1 & Sep/5 \\
10/MA & (Campillos, Almargen) & L1, LM6, ALM2 & Sep/5 \\
11/A & (Cuevas Bajas, Villanueva de Tapia) & L1, LM6, ALM2 & Sep/3 \\
\hline
\end{tabular}

Nota: $\mathrm{A}=$ activo; $\mathrm{MA}=$ muy activo; $\mathrm{NA}=$ no activo; $\mathrm{PA}=$ poco activo; $\mathrm{Mer}=$ meridional; $\mathrm{Sep}=$ septentrional.

Tabla 6. Resumen de las alineaciones y nudos (Cádiz / Málaga)

\begin{tabular}{|c|c|c|c|c|c|c|c|}
\hline \multirow{2}{*}{ Alineaciones } & \multicolumn{3}{|c|}{ Orden } & & \multicolumn{3}{c|}{ Orden } \\
\cline { 2 - 8 } & $\mathbf{2}$ & $\mathbf{3}$ & $\mathbf{4}$ & Nudos & $\mathbf{2}$ & $\mathbf{3}$ & $\mathbf{4}$ \\
\hline $17 / 12$ & $1 / 1$ & $9 / 7$ & $7 / 4$ & $20 / 12$ & $-/ 1$ & $8 / 5$ & $12 / 6$ \\
\hline
\end{tabular}

Se encontraron en C: I) 2 zonas compresivas: 1) Madina-SidoniaPaterna de Rivera (inmediaciones del nudo CV7 de $4^{\circ}$ orden, en Giletes); 2) Sierra de las Cabras (cerca del nudo CV4 de $3^{\text {er }}$ orden, en Sierra Vaquera); II) 2 importantes anomalías fluviales: 1) Verjer de la Frontera-Barbate (nudo CA7 de $4^{\circ}$ orden, en Barbate); 2) La Barca de la Horida (nudo CM1 de $3^{\text {er }}$ orden, en Torrecera). Estas zonas y anomalías están en los contactos de 4 grupos de bloques: B3-B9-B10, B1-B2, B1-B2-B16 y B8-B11-B12. El grado de actividad de esos son 9 bloques es: uno muy activo, dos activos, cinco poco activos y uno no activo, Estos conforman una figura geométrica triangular (área $\sim 600 \mathrm{~km}^{2}$ ). Esta zona activa del mesobloque I1 se inscribe, completamente, al N del PP de Primer Orden, justamente donde tiene una muy brusca inflexión al $\mathrm{S}$, hacia Tarifa $\left(\mathrm{Ks}_{\mathrm{s}}=0.33\right)$ y dista a $20 \mathrm{~km}$ al E de El Puerto de Santa María (figura 8).

En M se tienen: I) dos zonas compresivas: 1) Benmargosa-Comares (nudo MV6 de $4^{\circ}$ orden, en Villalón); 2) Sierra de las Salinas-Sierra de los Mimos-Sierra de Ortegicar (nudos MV3 y MM12, ambos de $3^{\text {er }}$ orden, y en Ronda y Embalse Gudaltela, respectivamente); II) una anomalía 
fluvial en Pizarra-Cartama (nudo MV4 de $4^{\circ}$ orden, en Cabras). Las zonas y la anomalía mencionadas aparecen en 4 grupos de bloques: B1-B6-B7, B1-B8-B9, B4-B5, y B1-B7-B10. La cantidad total de bloques involucrados es 9 (3 muy activos, 5 activos y 1 poco activo el mesobloque I1 y al $\mathrm{N}$ del PP de Primer Orden (figura 9). En este sentido, el PP y conforman un paralelogramo $\left(\sim 800 \mathrm{~km}^{2}\right)$, aproximadamente, paralelo a la costa, $25 \mathrm{~km}$ al $\mathrm{N}$ de Marbella. Los nudos y las deformaciones están en el W. En las figuras 8 y 9 se observan representaciones de la AS y de la densidad de epicentros, las cuales se corresponden bien en cuanto a dirección con las estructuras.

Desde el punto de vista de la sismoactividad y sobre la base de los trabajos de los autores, hay 4 segmentos en el S peninsular (C-M, Almería-Granada, Córdoba-Jaén y Murcia). Aseguramos que: 1) hay buena correspondencia entre la sismicidad y las estructuras delimitadas en la zona de Almería-Jaén; 2) en algunas de las morfoestructuras de Córdoba-Granada hay evidentes relaciones con la sismicidad, aunque en Córdoba los valores son menores que en Granada, donde existe un sector sismoactivo de $\sim 820 \mathrm{~km}^{2}$ entre las localidades de Beas de Granada-Lentejí-Loja; 3) las zonas de intersección de alineaciones en Murcia resultan ser muy activas (orden/cantidad) son: $2^{\circ} / 1,3^{\circ} / 12,4^{\circ} / 25,5^{\circ} / 38$; aunque su actividad es moderada, y de menor nivel que en Granada; 4) la correspondiente a C-M es también de menor nivel que en Granada, pero similar a la de Murcia; 5) las 5 isosistas indicadas para $\mathrm{C}$, en el punto 14 del epígrafe de Sismicidad, se relacionan con los nudos y alineaciones aquí determinados. Así tenemos que: A) con nudos se corresponden los terremotos de: A.1) San José del Valle ( $M=4.2 / \mathrm{h}=69 \mathrm{~km})$ en el nudo $\mathrm{CM} 1$, que está en la zona de mayor actividad; A.2.1) Puerto Serrano $(\mathrm{M}=3.7 / \mathrm{h}=29 \mathrm{~km})$ en el nudo CA4; A.2.2) Coripe ( $\mathrm{M}=3.4 / \mathrm{h}=5 \mathrm{~km})$ en el nudo CA4; B) con alineaciones se corresponden los terremotos de: B.1) Jerez de la Frontera $(\mathrm{M}=3.3$ / superficial) en el alineamiento L1; B.2) el terremoto de Prado del Rey $(\mathrm{M}=3.5 / \mathrm{h}=20 \mathrm{~km})$ en el alineamiento LC3.

Otras características de las 2 zonas activas de $\mathrm{C}$ y $\mathrm{M}$ son: 1) el alineamiento L1 enlaza a los nudos de $3^{\text {er }}$ orden CM1 (Cádiz) y MM12 (Málaga); 2) el 100\% de los bloques de $\mathrm{M}$ involucrados tienen actividad; mientras que en $\mathrm{C}$ son el $61 \%$; 3 ) la zona de $\mathrm{C}$ es hipsométricamente inferior a la de $\mathrm{M}$; 4) la zona de C $\left(36^{\circ} 10^{\prime} \mathrm{N} / 36^{\circ} 38^{\prime} \mathrm{N}, 5^{\circ} 41^{\prime} \mathrm{W} / 5^{\circ} 56^{\prime}\right.$ W) está localizada un poco más al $\mathrm{S}$ que la de $\mathrm{M}\left(36^{\circ} 44^{\prime} \mathrm{N} / 36^{\circ} 57^{\prime} \mathrm{N}\right.$,

Revista Geográfica de América Central No 55 ISSN 1011-484X, julio-diciembre 2015, pp. 169-198 
$\left.5^{\circ} 13^{\prime} \mathrm{W} / 4^{\circ} 24^{\prime} \mathrm{W}\right)$ y distan entre sí $\sim 40 \mathrm{~km}$; 5) las figuras geométricas de esas 2 zonas se corresponden muy bien con los trazos de las líneas de costa, respectivas, y los elementos morfológicos observados; 6) las áreas sismoactivas de $\mathrm{C}, \mathrm{M}$ y Granada tienen un valor del mismo orden, aunque el menor de ellos corresponde a $\mathrm{C}$.

Figura 7. Foto de un bloque en la provincia de Málaga

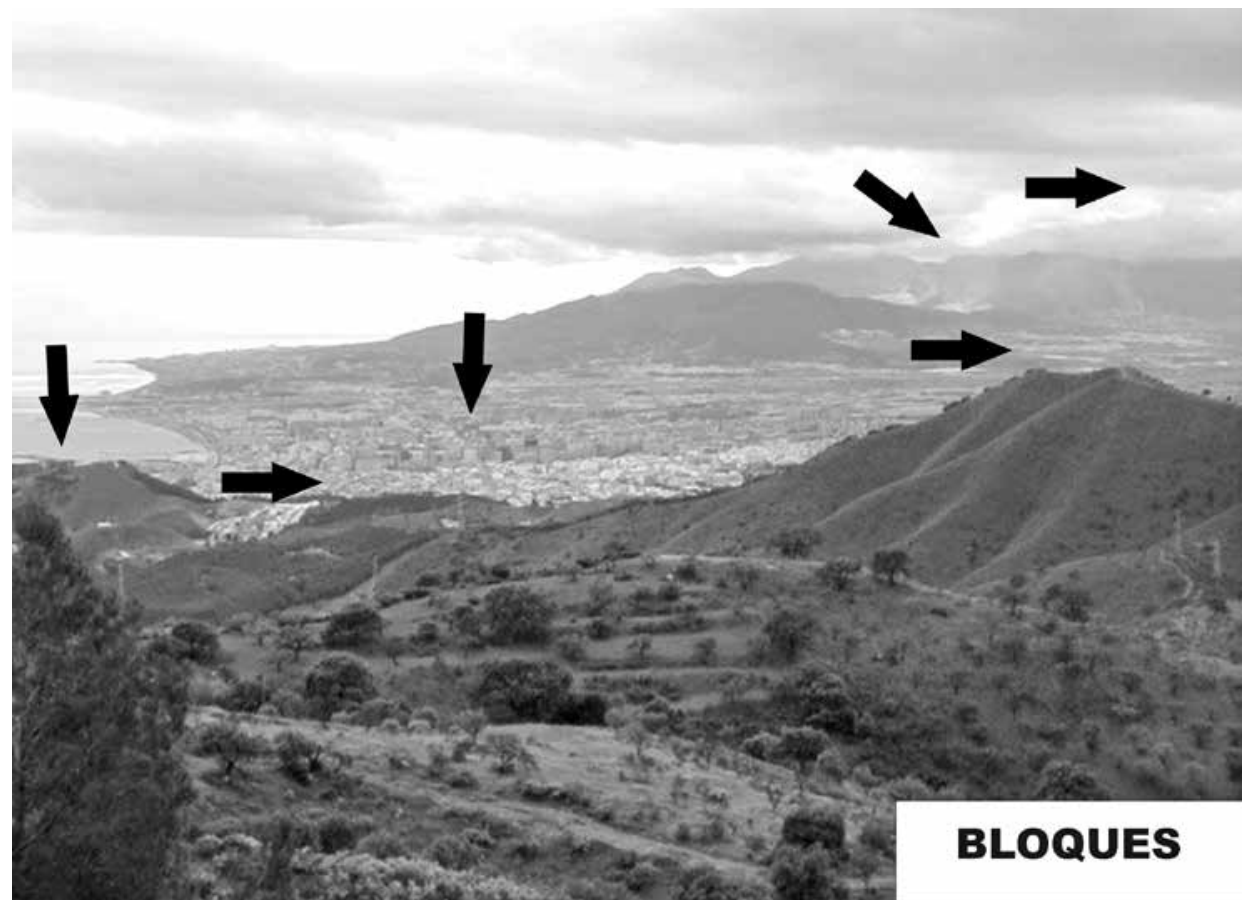

(Aparece la ciudad en un escalón altitudinal bajo y relativamente llano; se indican otros 5 niveles hipsométricos de la zona (flechas gruesas negras)). 
Mario Octavio Cotilla-Rodríguez, Diego Córdoba-Barba. Morphotectonics of the Cadiz-Malaga Segment, Southern Spain

Tabla 7. Datos de las alineaciones principales en Cádiz

\begin{tabular}{|c|c|c|c|c|}
\hline $\begin{array}{c}\text { Alineaciones } \\
\text { [Siglas] }\end{array}$ & Localidades & $\begin{array}{c}\text { Dirección / } \\
\text { Longitud (km) }\end{array}$ & $\begin{array}{c}\text { Orden / } \\
\text { Ks }\end{array}$ & Nudos [Siglas] (cantidad) \\
\hline L1 & Cádiz, Jerez de la Frontera & $\mathrm{NE} / 116$ & $2 / 0.85$ & $\begin{array}{l}\text { CA1, CA2, CA3, CA4, } \\
\text { CA5, CM1, CM2 (7) }\end{array}$ \\
\hline ALC3 & San Fernando & $\mathrm{NW} / 18$ & $3 / 0.80$ & CA4 (1) \\
\hline $\mathrm{LC} 2$ & Torrecera, Los Giletes & $\mathrm{NW} / 28$ & $3 / 0.90$ & CA9, CM1, CV3, CV7 (4) \\
\hline $\mathrm{LC} 3$ & San Enrique, Jimena de la Frontera & $\mathrm{N}-\mathrm{S} / 70$ & $3 / 0.76$ & $\mathrm{CA} 3, \mathrm{CV} 1, \mathrm{CV} 5, \mathrm{CV} 8$ (4) \\
\hline $\mathrm{LC} 4$ & Sierra Vaquera, Algodornales & $\mathrm{NW} / 18$ & $3 / 0.80$ & CA4 (1) \\
\hline LC5 & $\begin{array}{l}\text { Peñón de Zaframagón, Alcalá } \\
\text { del Valle }\end{array}$ & $\mathrm{NW} / 26$ & $3 / 0.91$ & CA5, CV6 (2) \\
\hline $\mathrm{LC} 12$ & $\begin{array}{l}\text { Jérez de la Frontera, Sierra } \\
\text { Gamuza }\end{array}$ & $\mathrm{NE} />30$ & $3 / 0.88$ & CA2 (1) \\
\hline $\mathrm{LC} 13$ & $\begin{array}{l}\text { Arcos de la Frontera, Sierra del } \\
\text { Calvario }\end{array}$ & $\mathrm{NE} />30$ & $3 / 0.87$ & CM2 (1) \\
\hline $\mathrm{LC} 14$ & Embalse de Bornos, La Cierva & $\mathrm{NE} / 10$ & $3 / 0.97$ & CA3 (1) \\
\hline $\mathrm{LC} 15$ & Sierra Vaquera, Cerro Galindo & $\mathrm{N}-\mathrm{S} / 10$ & $3 / 0.95$ & CA4 (1) \\
\hline $\mathrm{LC} 1$ & La Pedrosa, Algar & $\mathrm{E}-\mathrm{W} / 30$ & $4 / 0.85$ & CM2, CV4, CV5 (3) \\
\hline LC6 & $\begin{array}{l}\text { Embalse del Milagro, Cerro de } \\
\text { las Plata }\end{array}$ & $\mathrm{NW} / 40$ & $4 / 0.65$ & CA8, CV9 (2) \\
\hline $\mathrm{LC7}$ & $\begin{array}{l}\text { Chiclana de la Frontera, } \\
\text { Cortijo del Huerto }\end{array}$ & $\mathrm{NW} / 25$ & $4 / 0.98$ & CA6, CA9 (2) \\
\hline LC8 & Barbate, Los Barrios & NW-E-W/30 & $4 / 0.65$ & CA7, CV9 (2) \\
\hline LC9 & Cortijo de Isla Verde, Algar & $\mathrm{NE} / 30$ & $4 / 0.70$ & $\mathrm{CV} 2, \mathrm{CV} 4(2)$ \\
\hline $\mathrm{LC} 10$ & La Pedrosa, San José del Valle & $\mathrm{N}-\mathrm{S} / 28$ & $4 / 0.92$ & $\mathrm{CM} 2, \mathrm{CV} 3$ (2) \\
\hline $\mathrm{LC} 11$ & $\begin{array}{l}\text { Puerto Santa María, Cortijo del } \\
\text { Campanero }\end{array}$ & $\mathrm{NW} / 26$ & $4 / 0.98$ & CA1, CV7 (2) \\
\hline
\end{tabular}

Nota: $\mathrm{Ks}=$ coeficiente de sinuosidad.

Un resumen de las principales características morfotectónicas de las 4 regiones (Almería-Granada, C-M, Córdoba-Jaén y Murcia) está en la tabla 10. Esto permite comparar los datos y sostener que: 1) El segmento $\mathrm{C}-\mathrm{M}$ es morfotectónicamente similar a los otros 3 segmentos; 2) el segmento de Almería-Granada es el más activo de los 4; 3) se confirma que la macroregión C-Murcia es tectónicamente activa y representa muy bien las características de la zona de interacción entre las placas africana y del sector euroasiático en la PI. Además, se envidencia el nivel de la actividad en las regiones meridionales $\mathrm{y}$, se comprende que las zonas más activas son Almería y Granada.

190 Revista Geográfica de América Central No 55 ISSN 1011-484X, julio-diciembre 2015, pp. 169-198 
Tabla 8. Datos de las alineaciones principales en Málaga

\begin{tabular}{|c|c|c|c|c|}
\hline $\begin{array}{c}\text { Alineaciones } \\
\text { [Siglas] }\end{array}$ & Localidades & $\begin{array}{c}\text { Dirección / } \\
\text { Longitud (km) }\end{array}$ & $\begin{array}{c}\text { Orden / } \\
\text { Ks }\end{array}$ & Nudos [Siglas] (cantidad) \\
\hline $\mathrm{L} 1$ & Sierra de Molina, Sierra Llana & $\mathrm{E}-\mathrm{W} / 70$ & $2 / 0.89$ & MN8, MN9, MN12, MA7 (4) \\
\hline ALM1 & Charriana, Pizarra, Alora & $\mathrm{NW} / 40$ & $3 / 0.80$ & MM8, MV5, MV4 (3) \\
\hline ALM2 & Palenciana, Benamejí & E-W/60 & $3 / 0.87$ & MM11 (1) \\
\hline LM1 & Serrato, Sierra de las Salinas & $\mathrm{N}-\mathrm{NE} / 80$ & $3 / 0.71$ & MM12, MV2, MV3 (3) \\
\hline LM3 & Torre del Mar, Vélez-Málaga & $\mathrm{NE} / 70$ & $3 / 0.86$ & MN9, MV3 (2) \\
\hline LM5 & Sierra de las Cumbres, Zahara & $\mathrm{NW} / 30$ & $3 / 0.91$ & MV3 (1) \\
\hline LM6 & $\begin{array}{l}\text { Lomas de Chapera, Sierra de } \\
\text { las Nieves }\end{array}$ & $\mathrm{NE} / 32$ & $3 / 0.94$ & MN8, MN10 (2) \\
\hline LM8 & Huerta del Río, Cuevas Bajas & $\mathrm{N}-\mathrm{S} / 20$ & $3 / 0.81$ & MA7, MN11(2) \\
\hline LM2 & Marbella, Sierra Real & $\mathrm{N}-\mathrm{NW} / 20$ & $4 / 0.88$ & MN10, MN11 (2) \\
\hline LM4 & Tolox & $\mathrm{E}-\mathrm{W} / 30$ & $4 / 0.93$ & MN10, MN11, MV4 (3) \\
\hline LM7 & Santa Rosa, Almogía & $\mathrm{NE} / 25$ & $4 / 0.79$ & MV5, MV6 (2) \\
\hline LM9 & Gaucín, Sierra de la Hidalga & $\mathrm{NE} / 40$ & $4 / 0.88$ & MN10, MV2 (2) \\
\hline
\end{tabular}

Nota: $\mathrm{Ks}=$ coeficiente de sinuosidad.

Los Grupos II y III mencionados en el punto 12 del epígrafe de Sismicidad, caen fuera de la zona de estudio (C-M); pero el Grupo I se relaciona con: 1) el Golfo de $\mathrm{C}$, donde están las alineaciones A1 y L1, y los bloques 1 y 15 de C; 2) el tramo de Ubrique-Carmona está al E del nudo CV5, donde las Sierras de Ubrique-de las Viñas-de Caillo-del Endrina (pertenecientes a M) y a $18 \mathrm{~km}$ al E del nudo NV4 (de la zona activa de C); 3) el segmento de M-Adra, donde se distingue el bloque 5 de $\mathrm{M}$, que es muy activo.

Por último, se confirma que los Ks (de la costa / del PPPO) son muy diferentes en las partes septentrional $(0.88$ / 0.81) y meridional $(0.62$ / 0.57$)$ del megabloque PI. También, las cantidades de UT menores difieren: a) parte septentrional (bloques $=182$, microbloques $=135$, nanobloques $=293$ ); $b$ ) parte meridional (bloques $=131$, microbloques $=348$, nanobloques $=1,178$ ). Empleando las relaciones entre cada par de estas 3 UT se tienen los siguientes valores: Bloques septentrionales / Bloques meridionales=1,34; Microbloques septentrionales / Microbloques meridionales $=0.84$; y Nanobloques septentrionales / Nanobloques meridionales $=0.24$. Se aprecia un cambio, muy drástico en los 2 rangos inferiores (micro y nanobloques), los cuales entendemos que son el resultado de la actividad convergente actual en la parte meridional de la PI. Así, se puede asegurar que los datos morfotectónicos se corresponden con la dinámica actual del territorio. 
Mario Octavio Cotilla-Rodríguez, Diego Córdoba-Barba. Morphotectonics of the

Cadiz-Malaga Segment, Southern Spain

Tabla 9. Resumen de las intersecciones principales en Cádiz y Málaga

\begin{tabular}{|c|c|c|c|}
\hline \multicolumn{2}{|l|}{ Cádiz } & \multicolumn{2}{|c|}{ Málaga } \\
\hline $\begin{array}{l}\text { Nudos/Orden/ } \\
\text { Denominación }\end{array}$ & $\begin{array}{c}\text { Compuesto por las } \\
\text { alineaciones }\end{array}$ & $\begin{array}{l}\text { Nudos/Orden/ } \\
\text { Denominación }\end{array}$ & $\begin{array}{c}\text { Compuesto por las } \\
\text { alineaciones }\end{array}$ \\
\hline CA1/3/Puerto Santa María & L1 & MM1/2/Cuevas Bajas & LM8, ALM2 \\
\hline CA2/3/Jerez de la Frontera & L1-LC2-LC12 & MV2/3/Ermita del Rosario & LM1, LM9 \\
\hline CA3/3/Embalse de Bornos & L1-LC3-LC14 & MV3/3/Ronda & LM1, LM5 \\
\hline CA4/3/Sierra Vaquera & L1-LC4.LC15 & MV8/3/Sierra Llana & L1, LM6, ALM1 \\
\hline CA5/3/Zaframagón & L1-LC5 & MM9/3/Embalse Gaitane & L1, LM3, LM8 \\
\hline CA9/3/Benalup-Casas Viejas & ALC2,ALC3,LC2 & MM12/3/Embalse Gudaltela & L1, LM8 \\
\hline CM1/3/Torrecera & L1-LC2 & MA7/4/Guadaltela & L1, LM8 \\
\hline CM2/3/La Pedrosa & L1-LC2-LC13 & MN10/4/Huerta del Río & LM2, LM4, LM6 \\
\hline CA6/4/Chiclana de la Frontera & ALC3-LC7 & MN11/4/Sierra de las Nieves & LM2, LM4, LM6, LM9 \\
\hline CA7/4/Barbate & ALC2-LC8 & MV4/4/Villalón & LM4, ALM1 \\
\hline CA8/4/Embalse del Mlagro & ALC2-LC6 & MV5/4/Campanillas & LM4, LM7 \\
\hline CV1/4/Sierra Almenara & LC3-LM6 (Málaga) & MV6/4/Cabras & LM3, LM7 \\
\hline CV2/4/Momia & LC9-ALC2 & & \\
\hline CV3/4/Covatillas & LC2-LC10 & & \\
\hline CV4/4/Algar & LC1-LC9 & & \\
\hline CV5/4/Fátima & LC1-LC3 & & \\
\hline CV6/4/Olvera & $\mathrm{LC} 5, \mathrm{LC} 23$ & & \\
\hline CV7/4/Los Giletes & LC2-LC11 & & \\
\hline CV8/4/Cortijo del Lobo & LC3-ALC2 & & \\
\hline CV9/4/Los Barrios & LC6-LC8 & & \\
\hline
\end{tabular}

192 Revista Geográfica de América Central No 55 ISSN 1011-484X, julio-diciembre 2015, pp. 169-198 
Figura 8. Sistemas de bloques de Cádiz
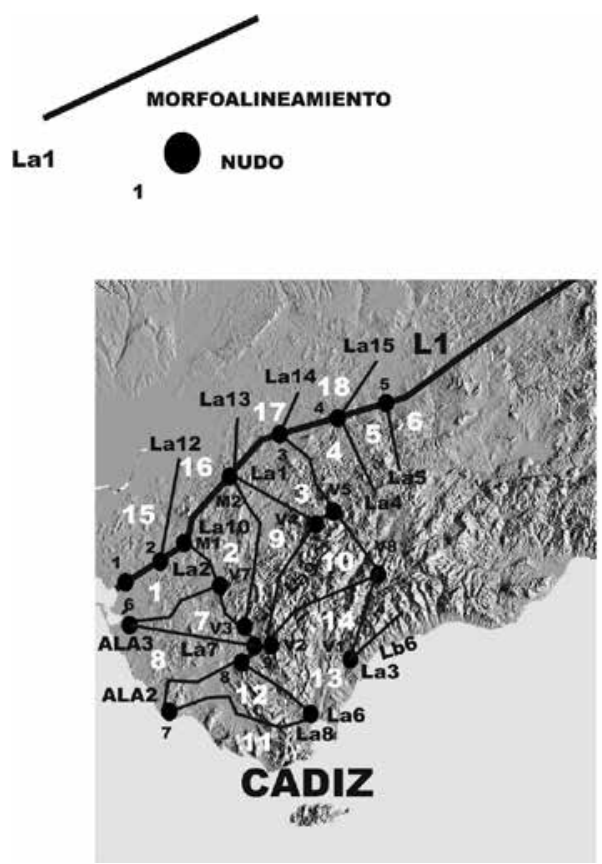

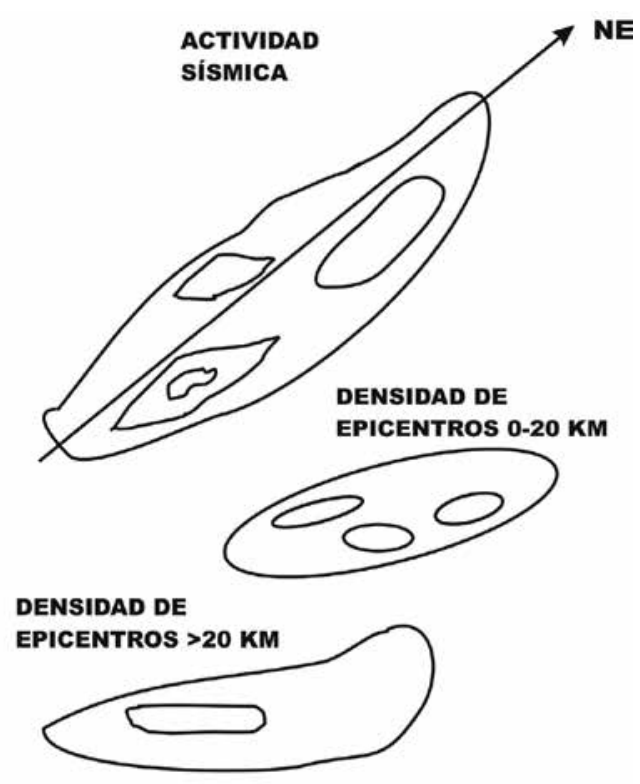

Se representan: 1) los bloques con números blancos (ver Tablas 3A y 4A); 2) los morfoalineamientos con líneas negras (ver Tabla 8); 3) los nudos con círculos negros (ver Tabla 9). Se dan las representaciones simplificadas de la Actividad Sísmica y la Densidad de Epicentros (para 2 rangos de profundidad). 
Mario Octavio Cotilla-Rodríguez, Diego Córdoba-Barba. Morphotectonics of the Cadiz-Malaga Segment, Southern Spain

Figura 9. Sistemas de bloques de Málaga
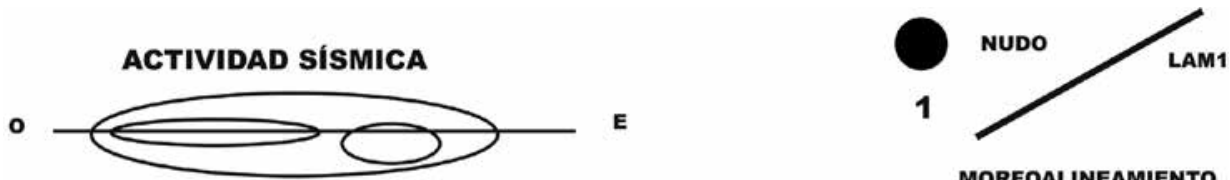

MORFOALINEAMIENTO

\section{DENSIDAD DE EPICENTROS 0-25 KM}

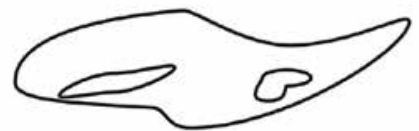

\section{DENSIDAD DE EPICENTROS} $>25 \mathrm{KM}$
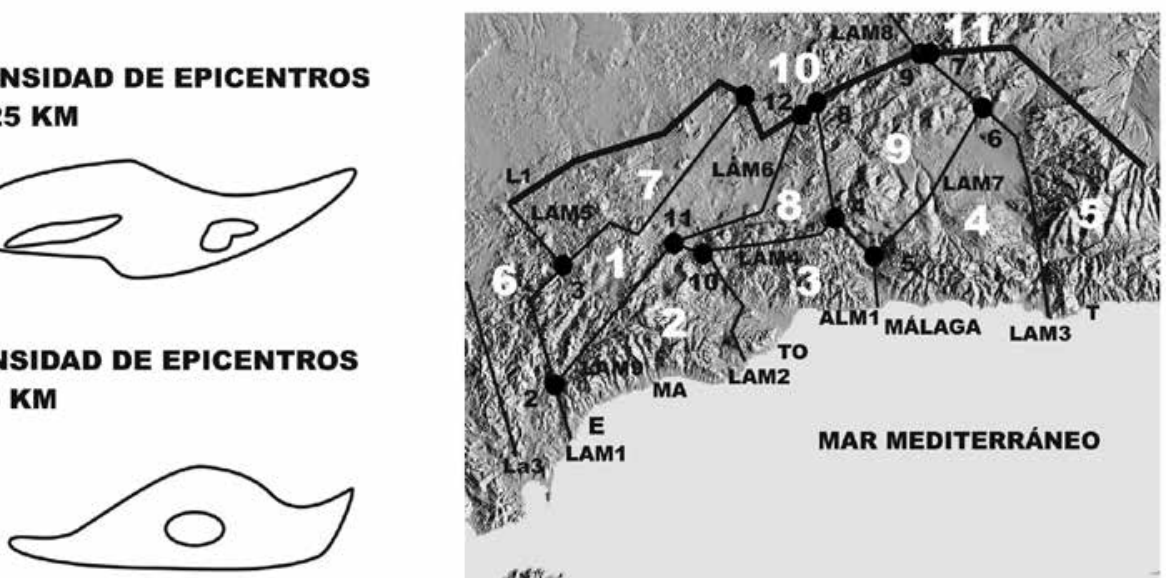

4\$口

Se representan: 1) los bloques con números blancos (ver Tablas 3A y 5A); 2) los morfoalineamientos con líneas negras (ver Tabla 8); 3) los nudos con círculos negros (ver Tabla 9). Se dan las representaciones simplificadas de la Actividad Sísmica y la Densidad de Epicentros (para 2 rangos de profundidad).

Tabla 10. Resumen de las Unidades Territoriales del sur de la Península Ibérica

\begin{tabular}{|c|c|c|c|c|c|c|c|}
\hline Región & $\begin{array}{c}\text { Macro/ } \\
\text { Meso }\end{array}$ & Bloques & Micro & Nano & Alinea & Nudos & Escarpes \\
\hline $\mathrm{Al}-\mathrm{Gr}$ & $2 / 5$ & $\mathrm{Al}=13$ & $\mathrm{Al}=36$ & $\mathrm{Al}=110$ & $\mathrm{Al}=29$ & $\mathrm{Al}=26 /$ & $\mathrm{Al}=10$ \\
& $2 / 2$ & $/ \mathrm{Gr}=9$ & $/ \mathrm{Gr}=32$ & $/ \mathrm{Gr}=58$ & $/ \mathrm{Gr}=25$ & $\mathrm{Gr}=46$ & $/ \mathrm{Gr}=17$ \\
$\mathrm{C}-\mathrm{M}$ & $\mathrm{C}=18$ & $\mathrm{C}=18$ & $\mathrm{C}=90$ & $\mathrm{C}=17$ & $\mathrm{C}=20$ & $\mathrm{C}=4$ \\
& $2 / 4$ & $\mathrm{M}=11$ & $\mathrm{M}=26$ & $/ \mathrm{M}=112$ & $/ \mathrm{M}=12$ & $/ \mathrm{M}=12$ & $/ \mathrm{M}=7$ \\
$\mathrm{Co}-\mathrm{J}$ & & $\mathrm{Co}=9$ & $\mathrm{Co}=16$ & $\mathrm{Co}=51$ & $\mathrm{Co}=16$ & $\mathrm{Co}=28$ & $\mathrm{Co}=7$ \\
& $2 / 3$ & 47 & 160 & 500 & 15 & $/ \mathrm{J}=57$ & $/ \mathrm{J}=9$ \\
$\mathrm{Mu}$ & & 131 & 348 & 1,178 & 166 & 206 & 63 \\
\hline
\end{tabular}

Nota: Al-Gr= Almería-Granada; C-M= Cádiz-Málaga; Co-J= Córdoba-Jaén; $\mathrm{Mu}=$ Murcia; Macro= macrobloque; $\mathrm{Meso}=$ mesobloque; $\mathrm{Micro}=$ microbloque; $\mathrm{Nano}=$ nanobloque; Alinea= alineamiento.

194 Revista Geográfica de América Central No 55 ISSN 1011-484X, julio-diciembre 2015, pp. 169-198 
Figura 10. Esquemas de las dos áreas sismoactivas

el segmento Cádiz-Málaga

(ver Figura 1C)

$\Delta$

\section{MESOBLOQUE I}

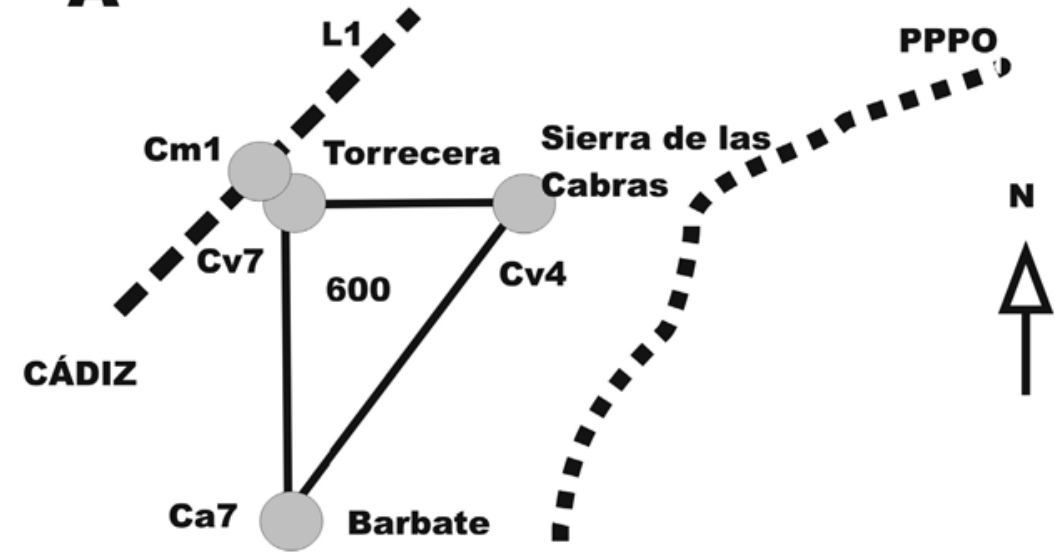

TARIFA

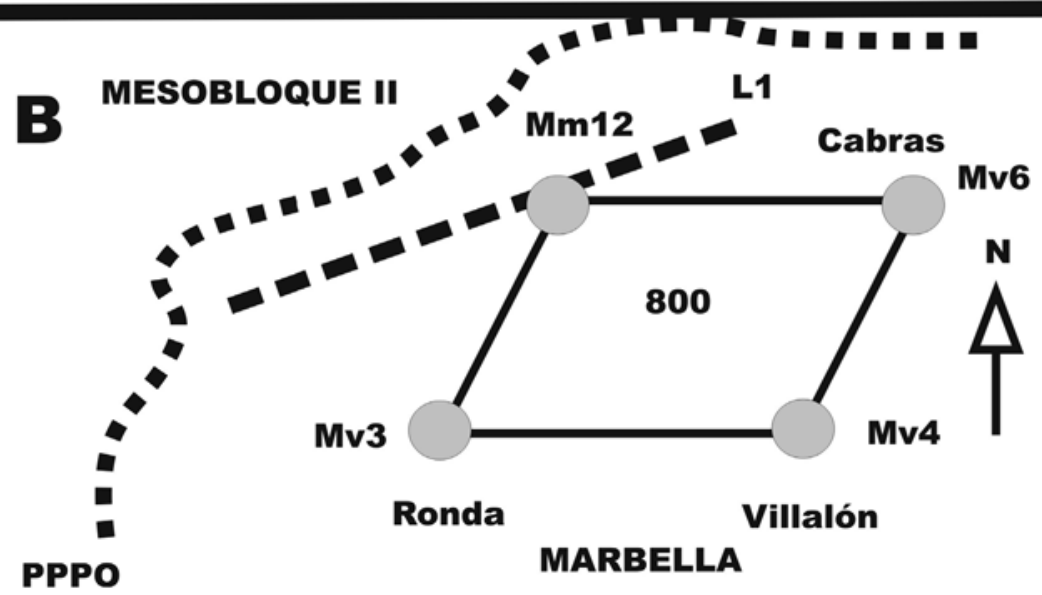

Cádiz. Aparecen: 1) cuatro nudos (CA7, CM1, CV4 y CV7); 2) un alineamiento (L1); 3) el Parteaguas Principal de Primer Orden (PPPO); 4) el valor del área en $\mathrm{km}^{2}$.

Málaga. Aparecen: 1) cuatro nudos (MM12, MV3, MV4 y MV6); 2) un alineamiento (L1); 3) el Parteaguas Principal de Primer Orden (PPPO); 4) el valor del área en $\mathrm{km}^{2}$. 


\section{Agradecimientos}

A la Facultad de CC Físicas y en particular al Departamento de Geofísica y Meteorología, Universidad Complutense de Madrid, por la utilización de los recursos informáticos y bibliográficos. Este trabajo fue financiado en parte con los proyectos: TOPOIBERIA (CSD2006/0041) y TSUJAL (CGL2011-29474-C02-01). Las figuras las preparó Amador García Sarduy. Los evaluadores y filóloga de la Revista han realizado sugerencias que han mejorado la exposición del trabajo.

\section{Referencias}

Alekseevskaya, M., Gabrielov, A., Gelfand, I., Gvishiani, A. y Rantsman, E. (1977). Formal morphostructural zoning in mountain territories. Journal Geophys, 43, 227-233.

Assinovskaya, B.A. y Solovyev, S.L. (1994). Definition and description of the sources zone of potencial earthquakes in the Barents Sea. Physics of the Solid Earth, 29(8), 664-675.

Buforn, E., Bezzeghoud, M., Udías, A. y Pro, C. (2004). Seismic sources on the Iberia-African Plate Boundary and their tectonic implications. Pure appl. geophys, 161, 623-646.

Buforn, E., Sanz de Galdeano, C. y Udías, A. (1995). Seismotectonics of the Ibero-Maghrebian region. Tectonophysics, 248, 247-261.

Calvert, A., Sandool, E., Seber, D., Barazangi, M, Roeker, S., Mourabit, T., Vidal, F., Alguacil, G. y Jabour, N. (2000). Geodynamic evolution of the lithosphere and upper mantle beneath the Alboran region of the western Mediterranean: Contraints from travel time tomography. Journal Geophys. Res., 105, 10.871-10.898.

Capote, R., Insua, J.M., Martínez-Díaz J.J., Martín-González F. y Tsige M. (2002). La serie de Cártama: Pliegue con actividad reciente en las Béticas Occidentales (Hoya de Málaga). Geogaceta, 31, 135-138.

Cotilla M.O. y Córdoba D. (2009). Morfotectónica de Murcia, España. Revista Geográfica, 146, 77-110.

Cotilla, M.O. y Córdoba, D. (2007). A morphotectonic study of the Central System, Iberian Península. Russian Geology and Geophysics, 48(4), 378-38.

Cotilla, M.O. y Córdoba, D. (2004A). Morphotectonics of the Iberian Peninsula. Pure appl.geophys, 42(4), 589-602.

Revista Geográfica de América Central No 55 ISSN 1011-484X, julio-diciembre 2015, pp. 169-198 
Cotilla, M.O. y Córdoba, D. (2004B). Present geomorphological characteristics of Alboran islet and surroundings, Spain: a diagnosis. Geogr. Fis. Dinam. Quat., 27, 3-19.

Cotilla, M.O. y Córdoba, D. (2003). Caracterización morfotectónica de Galicia, España. Revista Geofisica, 58, 5-56.

Cotilla, M.O. y Córdoba, D., (en prensa). Delimitación de unidades morfotectónicas en el sector Almería-Jaén, España. Revista Geológica Colombiana. En prensa.

Cotilla, M.O., Córdoba, D. y Herraiz M. (2004). Main morphotectonic characteristics of Asturias, Spain. Geofisica International, 44(1), 65-101.

Cotilla, M.O., Córdoba, D. y Sánchez, F. (2003). Morphotectonic study of two regions in the Centre-South segment of Spain: Córdoba and Granada. Geotectonics, 47(3), 215-240.

Galindo-Zaldívar, J., González-Lodeiro, F. y Jabaloy, A. (1993). Stress and paleostress in the Betic-Rif cordilleras (Miocene to the present). Tectonophysics, 227, 105-226.

Gatinsky Yu., G. y Rundquist, D.V. (2004). Geodynamics of Eurasia: Plate tectonics and block tectonics. Geotectonics, 38(1), 1-16.

Gorshkov, A.I., Kuznetsov, I.V., Panza G.F. y Soloviev A.A. (2000). Identification of future earthquake sources in the Carpatho-Balkan orogenic belt using morphostructural criteria. Pure appl. geophys., 157, 79-95.

Gurría, E. y Mezcua, J. (2000). Seismic tomography of the crust and the lithospheric mantle in the Betic Cordillera and Alboran Sea. Tectonophysics, 329, 99-119.

Henares, J. y López, C. (2001). Catálogo de mecanismos focales del área Ibero-Mogrebi. (Editores: C. López Casado). Universidad de Granada, Instituto Andaluz de Geofísica y Prevención de Desastres Sísmicos, Dpto. de Física Teórica y del Cosmos, 208.

Herráiz M., De Vicente G., Lindo R., Giner J., Simón J.L., Gómez-Casado J.M., Vadillo O., Rodríguez-Pascuas M., Cicuendez, J.I., Casas, A., Cabañas, L., Rincón, P., Cortés, M., Ramírez, M. y Lucini, M. (2000). The recent (Upper Miocene to Quaternary) and present tectonic stress distribution in the Iberian Peninsula. Tectonics, 19(4), 762-786. 
Instituto Andaluz de Geofísica, IAG (2002). Recuperado de: http://www. ugr.es/ iag/tensor.

Martínez, J. M. (2003). Sismicidad histórica de la Península Ibérica. Revista Física de la Tierra, 15, 13-28.

Mezcua, J. y Rueda, J. (2006). Sismicidad, sismotectónica y evolución geodinámica de la Península Ibérica. Revista Física de la Tierra, 18, 137-155.

Mezcua, J., Rueda, J. y García, R.M. (2004). Reevaluation of historic earthquakes in Spain. Seismol. Res. Lett., 75, 75-81.

Negredo A., Bird P., Sanz de Galdeano, C. y Buforn, E. (2002). Notectonic modeling of the Ibero-Maghrbian region. Journal Geophys. Res., 107, 10,1-10,14.

Rantsman, E. Ya. (1979). Sites of earthquakes and morphostructures of mountain countries. Editorial Nauka, Moscú, 171.

Reicherter, K.R. y Peters G. (2005). Neotectonic evolution of the Central Betic Cordilleras (Southern Spain). Tectonophysics, 405 (pp. 191-212).

Sanz de Galdeano, C., López, C., Delgado J. y Peinado, M.A. (1995). Shalow seismicity and active faults in the Betic Cordillera. A preliminary approach to seismic sources associated with specific faults. Tectonophysics, 248, 293-302.

Soto, J.I. y Manzano, F.J. (2002). Geometría y cinemática de las fallas recientes en el margen septentrional del Mar de Alborán (sector de Maro, provincia de Málaga). Geogaceta, 31, 139-142.

Zhidkov, M.P., Rotvain, I.M. y Sadowskiy, A.M. (1975). Pronóstico sobre las áreas más probables de ocurrencia de los terremotos más fuertes, IV. Papers of Seismology, 8, 53-70. 